\title{
Performance Enhancing of Galloping-based Piezoelectric Energy Harvesting by Exploiting 1:1 Internal Resonance of Magnetically Coupled Oscillators
}

\author{
Wan Sun \\ Jiangsu University \\ Canzhi Guo \\ Jiangsu University \\ Guanggui Cheng \\ Jiangsu University \\ Shangwen He \\ Zhengzhou University \\ zhaorui yang ( $\nabla$ zryang@zzu.edu.cn ) \\ Zhengzhou University \\ Jianning Ding \\ Jiangsu University
}

\section{Research Article}

Keywords: Internal resonance, Multiple time scale, Frequency synchronization, Nonlinear force coupling, Wind energy harvesting

Posted Date: November 3rd, 2021

DOI: https://doi.org/10.21203/rs.3.rs-1022013/v1

License: (c) (i) This work is licensed under a Creative Commons Attribution 4.0 International License. Read Full License 
1 Performance enhancing of galloping-based piezoelectric energy

2 harvesting by exploiting 1:1 internal resonance of magnetically coupled oscillators

4 Wan Sun ${ }^{\mathrm{a}, \mathrm{b}}$, Canzhi Guo ${ }^{\mathrm{a}, \mathrm{b}}$, Guanggui Cheng ${ }^{\mathrm{a}, \mathrm{b}}$, Shangwen $\mathrm{He}^{\mathrm{b}}$, Zhaorui Yang ${ }^{\mathrm{b} *}$, Jianning Ding a,b*

5 a Institute of Intelligent Flexible Mechatronics, Jiangsu University, Zhenjiang 212013, P.R. China

6 b Jiangsu Collaborative Innovation Center of Photovoltaic Science and Engineering, Changzhou

7 University, Changzhou 213164, P.R. China

$8{ }^{\mathrm{c}}$ School of Mechanics and Safety Engineering, Zhengzhou University, Zhengzhou 450001, P.R. China

$12 *$ Corresponding Author

13 E-mail address:

14 zryang@zzu.edu.cn (Zhaorui Yang)

15 dingjn@ujs.edu.cn (Jianning Ding) 


\section{Abstract}

In this study, we introduced 1:1 internal resonance in a magnetically coupled 2-degree-of-freedom

(2-DOF) galloping-based piezoelectric energy harvester to improve the energy harvesting efficiency.

The governing equations for the proposed magnetically coupled aero-electro-mechanical system considering the effect of oscillating wake are established using the extended Hamilton principle, and the method of multiple scale is exploited to obtain approximate analytical solutions. Parameter sweeping numerical calculation is conducted to validate the analytical prediction through comparing with analytical solutions, and the results show a good matching between them. In addition, we investigated the systematic dynamic behaviors under a pure oscillating wake induced 1:1 internal response. Typical nonlinear characteristics such as jump, hysteresis, frequency synchronization under varying design parameters appear in the present system. Especially, cusp bifurcation and synchronization regime of oscillating wake coupled nonlinear oscillator in $\eta-\Theta_{w}$ plane and $\sigma-\Theta_{w}$ plan. With an adoption of magnetic force, chaos happens as the gap distance decreases smaller than $5 \mathrm{~mm}$, and a frequency lock-in phenomenon can be strengthened through adjusting the magnet distance. In the perspective of output performance, both of the voltage and power output results shows that the exploiting of 1:1 internal resonance can significantly improve the output performance under a suitable magnet distance.

Keyword: Internal resonance; Multiple time scale; Frequency synchronization; Nonlinear force coupling; Wind energy harvesting. 


\section{Introduction}

With the dramatic increasing of energy consumption due to the rapid industrial development, a

series of environmental pollution problems have been continually arisen over the past few decades. It is

4 anticipated that the development of renewable and sustainable energy is the trend of human development

5 in the future, and this viewpoint is becoming the consensus all over the world. Energy harvesting from

6 the surrounding environment as an alternative of renewable energy has been receiving increasing interest

7 in recent few decades. In particular, the recent rapid developments of wireless sensor networks

8 necessitate some new demands on self-powering technologies such as portability, small-scale, high

9 efficiency, environmental adaptability, which promotes numerous researchers devoting their efforts in

10 developing various energy harvesting technologies.

11 In accordance with the operation environment of the sensor nodes and electric devices, various

12 forms of wasted energy sources such as wind [1], mechanical [2, 3], thermal, solar, and chemical sources

13 can be selected and converted into electric energy for the electric consumption devices. Wind energy, as

14 one of the most common wasted energy sources, which has abundant free utilization value. A large

15 amount usage of wind energy has been applied to the traditional rotating wind turbine $[4,5]$ for

16 commercial power supplies because of its high output and high efficiency in wind energy conversion.

17 On the other hands, wind turbine is difficult to meet the requirements of self-power supply of small-

18 scale wireless sensors that need a compact structural integration of the power supply components and

19 target small-scale electric device. Therefore, the instinct shortage of the wind turbine applying in small-

20 scale electric devices accelerated the development of the studies on compact wind energy harvesting 
1 system based on flow induced vibrations such as vortex-induced vibration (VIV), galloping, flutter, and

2 wake galloping. Among them, galloping phenomenon is a representative flow-induced vibration, which

3 happens when the structural system loses its stability. The galloping phenomenon is broadly applied in a

4 small-scale wind energy harvesting system because it usually exhibits a large oscillation amplitude and

5 wide operating wind velocity range compared to VIV, flutter and wake galloping [6-8].

6 The wind energy can be converted into electric energy through three typical transduction

7 mechanisms of electromagnetic, electrostatic, and piezoelectric. Among them, the piezoelectric

8 mechanism is the most popular owing to its high energy density and easiness of application [8-10]. In

9 the last decade, a variety of piezoelectric wind energy harvesters (PEH) have been studied from the basis

10 mechanisms of flow induced vibration to the technologies of improving energy conversion efficiency

11 [11-15]. Among various aerodynamic phenomenon-based wind energy harvesters, galloping-based

12 piezoelectric wind energy harvester (GPEH) exhibits an excellent performance compared to the others

13 because of its larger amplitude response and wider operational wind range. A general GPEH is

14 composed of a cantilever beam attached with piezo patch and a cross-section asymmetric bluff body

15 fixed on its free end. To further improve energy harvesting efficiency and reduce the critical wind speed,

16 some researchers have been devoting their efforts on optimizing the cross-section of bluff body [16-21],

17 exerting synergy effect by combining aerodynamic phenomena (VIV, galloping) [22-25] and increasing

18 the degrees of freedom (DOF) [26, 27]. Lan et al. [26] proposed lumped parameter models for two

19 structural configurations with different bluff bodies under aerodynamic force. They analytically and 
numerically compared the output performances between the proposed configurations which shows that

2 the second configuration with the wind flow loaded on the outer bluff body can easily and remarkably

3 reduce the critical wind speed and improve the output power. In addition, $\mathrm{Hu}$ et al. [7] recently proposed

4 a comb-like beam (CombBeam) based GPEH, which consists of several parasitic beams mounted on a

5 conventional cantilever beam. This structure is regarded as a multiple-degree-of-freedom (MDOF)

6 system. They showed that the MDOF CombBeam-based GPEH was superior to the conventional beam

7 GPEH in reducing the cut-in wind speed from $2.24 \mathrm{~m} / \mathrm{s}$ to $1.96 \mathrm{~m} / \mathrm{s}$ and enhancing the power for about

$8 \quad 171.2 \%$ with an optimal resistance under a specific wind speed of $3 \mathrm{~m} / \mathrm{s}$. Starting from such kinds of

9 linear 2-DOF GPEH, an exploiting of external nonlinear force in a flow induced vibration energy

10 harvester was also considered in some works to further enhance the energy harvesting performance.

11 Wang et al. [28] proposed a double-beam piezo-magneto-elastic nonlinear wind energy harvester, in

12 which two piezoelectric cantilever beams attached to prism-like bluff bodies employing repulsive

13 magnets were fixed vertically and oriented to face each other. Most recently, Sun et al. [6] investigated a

14 magnetically coupled GPEH in a tandem configuration to improve the energetic performance, which

15 consists of two elastic structures with two bluff bodies and a pair of repulsive magnets installed for the

16 generation of mutual interaction between them. This work showed that a maximum overall average

17 output power of $8.09 \mathrm{~mW}$ and power improvement rate of $65 \%$ were obtained compared to the case

18 without magnetic interaction under an optimal magnetic gap distance.

19 However, to the best of our knowledge, few study has reported on consideration of the effects of 
1 the natural frequency ratio between the oscillators to the overall output performance in a two or multiple

2 DOF GPEH. Up till now, some systematic studies on the internal resonance have been carried out on a

3 general 2-DOF PEH in the previous works. In these previous works [29-32], the dynamical behaviors

4 show dramatical changes when one of the natural frequencies is close to the other where internal

5 resonance happens. Internal resonance has been extensively reported in external forced vibration energy

6 harvesting system for an output performance enhancement [33,34], which usually appears when the

7 ratio between two natural frequencies of a given system is close to an integer and various interesting

8 phenomena such as frequency or (and) lock-in (synchronization), hysteresis, chaotic behaviors can be

9 resulted in. Inspired by these work on internal resonance in a 2-DOF PEH, this study tried to exploit 1:1

10 internal resonance in a development of magnetically coupled galloping-based piezoelectric energy

11 harvester (MCR- GPEH) for a significant output performance improvement.

This study proposed a lumped parameter model for the MCR-GPEH considering the effect of

13 oscillating wake using Hamilton principle, and a series of comprehensive parametric studies have been

14 analytically and numerically conducted to predict the evaluation of the symmetric dynamic behavior

15 when the internal resonance happens. This work provides a general framework and design guidance for

16 a high efficiency wind energy harvester by exploiting 1:1 internal resonance through controlling the

17 distance. Both of the numerical and analytical results showed that 1:1 internal resonance wind energy

18 harvesting system is expected to be a promising alternative in the development of high-efficiency

19 galloping-based wind energy harvesting system. 


\section{Dynamic modeling}

\subsection{Conceptual description} 11 in the previous studies.

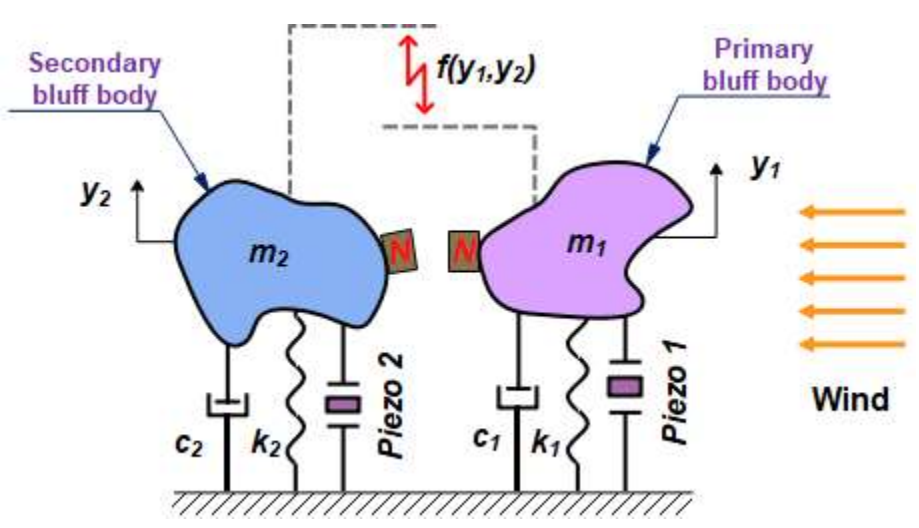

Fig. 1 Schematic of the magnetically coupled 2-DOF galloping-based piezoelectric energy harvester.

phenomenon is generally designed with a single cantilever beam and a bluff body fixed on its free end.

The wind energy can be converted into structural vibration energy, and then into electric energy through the piezoelectric transducer, which has been thoroughly investigated in various aspects such as fundamental theory construction, novel structure design and aerodynamic energy conversion mechanism harvester to increase the energy harvesting efficiency through double-steps energy extraction from the incoming wind flow using the tandem arrangement of the bluff bodies as shown in Fig. 1. The proposed structure is configured with two presumed arbitrary bluff bodies and individually connected to a fixture using elastic component. Considering the merits of galloping phenomenon in wind energy harvesting as 
1 introduced in Introduction section, the cross-section shape of the bluff bodies is designed as circular

2 asymmetric shape for aerodynamic instability. The primary bluff body is arranged in the front of the

3 secondary bluff body with a tandem configuration along the wind incoming direction. The wind flow

4 passing the primary bluff body generates wake flow that applies to the secondary bluff body and further

5 give rise to the variation of its dynamic behaviors. However, the oscillation of the secondary bluff body

6 has a negligible influence to the primary bluff body due to the directionality of the wake flow as

7 investigated in [35]. In this study, we exploited a pair of mutually repelling magnets, which are attached

8 to the inner side of each bluff body to strengthen the coupling effect of the dynamic behaviors of the two

9 bluff bodies to achieve synergistic effect.

10 This study focuses on the investigation of the overall output performance of the two individual

11 elastic structures under various natural frequency difference. The nonlinear characteristics induced by

12 the wind flow and magnet force lead to various nonlinear dynamic behaviors such as jump phenomenon,

13 limit cycle, frequency synchronization and catastrophe bifurcation. The variation of the natural

14 frequency difference between two weakly coupled mass-spring-damper system results in

15 synchronization and internal resonance which is beneficial to improve the wind energy harvesting

16 performance.

\subsection{Governing equations}

18 In this section, a mathematical modeling of the proposed magnetically coupled 2-DOF system is

19 derived to predict the dynamic behaviors and output voltage performance. The proposed system consists 
1 of two discrete mass-spring-damper components which are coupled with weak nonlinear magnetic force,

2 the governing equations can be formulated using the expended Hamilton's principle as follows:

$$
\int_{t_{1}}^{t_{2}}\left(\delta T_{t}-\delta \Pi_{t}+\delta W_{n c}\right) \mathrm{d} t=0
$$

4 where $t$ represents time, $\delta T_{t}$ and $\delta \Pi_{t}$ represent the variations of the total kinetic and potential energy 5 contained in this system, respectively, and $\delta W_{n c}$ is the virtual work done by the non-conservative forces.

6 The kinetic energy in the proposed system includes the translational and rotational kinetic energies of 7 the outer and inner bluff bodies. Thus, the total kinetic energy $T_{t}$ in the present system is given by

$$
T_{t}=\frac{1}{2} m_{1} \&+\frac{1}{2} m_{2} \&
$$

9 where the over-dot of a symbol denotes a derivative with respect to time $t ; m_{1}$ and $m_{2}$ denote the total

10 masses of the bluff bodies (primary, secondary) and the corresponding attached permanent magnets,

11 respectively; $y_{1}$ and $y_{2}$ denote the displacement of the primary and secondary bluff bodies.

13 electromechanical, mechanical, and magnetic potential energy. Subsequently, the total potential energy

$14 \Pi_{t}$ of the proposed system is expressed as follows:

$$
\Pi_{t}=U_{m a g}+\frac{1}{2} k_{1} y_{1}^{2}+\frac{1}{2} k_{1} y_{2}^{2}-\frac{1}{2} c_{p 1} \pi_{1}^{\&}(t)^{2}-\frac{1}{2} c_{p 2} \&_{2}^{\&}(t)^{2}-\theta_{e 1} k_{1}^{\&}(t) y_{1}-\theta_{e 2} z_{2}^{\&}(t) y_{2},
$$

16 where $k_{1}$ and $k_{2}$ represent the spring constants of the primary and secondary spring; $c_{p 1}$ and $c_{p 2}$

17 represent the capacitance of the primary and secondary piezo components; $\theta_{e 1}$ and $\theta_{e 2}$ represent the

18 piezoelectric coupling coefficients; $k_{1}^{\&}$ and $k_{2}^{\&}$ represent the flux linkage of the equivalent circuit of the 
1 piezoelectric components; $U_{\text {mag }}$ represents the potential energy from the magnetic field generated by

2 the two repulsive magnets, which can be described by the dipole-dipole model due to the small size

3 compared to the bluff body [36].

$$
U_{\text {mag }}=-\frac{\mu_{0}}{4 \pi}\left(\nabla \frac{\mathbf{m}_{B} \cdot \mathbf{r}_{B A}}{\left\|\mathbf{r}_{B A}\right\|_{2}^{3}}\right) \cdot \mathbf{m}_{A}=\frac{\mu_{0}}{4 \pi}\left[\frac{1}{\left\|\mathbf{r}_{B A}\right\|_{2}^{3}} \mathbf{m}_{B}-\frac{3\left(\mathbf{m}_{B} \cdot \mathbf{r}_{B A}\right)}{\left\|\mathbf{r}_{B A}\right\|_{2}^{5}} \mathbf{r}_{B A}\right] \cdot \mathbf{m}_{A},
$$

where $\mu_{0}$ is the vacuum permeability; $\mathbf{r}_{B A}$ is the vector from the center of the magnet A to the center of

6 the magnet B; and $\mathbf{m}_{A}$ and $\mathbf{m}_{B}$ are the magnetic moment vectors of the outer and inner magnets,

7 respectively. For a permanent magnet, the magnitude of the magnetization vector can be evaluated using

8 the magnet's residual flux density $B_{r}$, that is, $\bar{m}=B_{r} / \mu_{0}$. Under a series of mathematical manipulation,

9 the magnetic potential energy can be expressed as

10

$$
U_{m a g}\left(y_{1}, y_{2}\right)=-\frac{\bar{m}_{1} \bar{m}_{2} \bar{v}_{1} \bar{v}_{2} \mu_{0}}{4 \pi}\left(y_{12}{ }^{2}+D_{m}{ }^{2}\right)^{-1.5}-3 D_{m}{ }^{2}\left(y_{12}^{2}+D_{m}^{2}\right)^{-2.5} \text {, with } y_{12}=y_{1}-y_{2} \text {, }
$$

11 referring to [6]. where $\bar{m}_{1}$ and $\bar{m}_{2}$ are the magnitudes of magnetization vectors, and $\bar{v}_{1}$ and $\bar{v}_{2}$ are the

12 volumes of the primary and secondary magnets; and $D_{m}$ is the minimum gap distance between the

13 center points of the two magnets. Take the derivative with respect to $y_{12}$ on the magnetic potential

14 energy and then expand the resulting equation in a Taylor series at $y_{12}=0$, the magnetic force can be

15 obtained as

$$
F_{\text {mag }}= \pm\left(\mathrm{T}_{1} Y_{12}+\mathrm{T}_{3} Y_{12}{ }^{3}\right) \text {, with } \mathrm{T}_{1}=-\frac{12 \bar{m}_{A} \bar{m}_{B} \bar{v}_{A} \bar{v}_{B} \mu_{0}}{4 \pi D_{m}^{5}}, \mathrm{~T}_{3}=\frac{45 \bar{m}_{A} \bar{m}_{B} \bar{v}_{A} \bar{v}_{B} \mu_{0}}{4 \pi D_{m}^{7}}
$$




$$
\begin{aligned}
\delta W_{n c}= & F_{\mathrm{g} 1} \delta y_{1}+F_{g 2} \delta y_{2}+\theta_{1}^{w} \& \delta y_{2}+\theta_{2}^{w} \& \delta y_{1}+\delta y_{1}-c_{1} \& \delta y_{1}, \\
& -c_{2} \& \delta y_{2}-z_{1}(t) \delta \lambda_{2}(t) / R_{L 2}-\lambda_{2}^{\&}(t) \delta \lambda_{2}(t) / R_{L 2}
\end{aligned}
$$

2 where $R_{L 1}$ and $R_{L 2}$ represent the load resistances for the primary and secondary external circuit,

3 respectively; $c_{1}$ and $c_{2}$ represent the damping constants; $\theta_{i}^{w}$ denote the wake force coefficients, and we

4 assumed they are both equal to $\Theta_{w} ; F_{g 1}$ and $F_{g 2}$ are the aerodynamic transverse force acting on the

5 bluff bodies, which can be expressed as [23]

$$
F_{g i}=n_{i} U_{i}^{2} C_{g i} \text { with } n_{i}=0.5 \rho_{\mathrm{a}} D H_{i},
$$

7 where the indices $i=1,2$ represent the corresponding symbols for primary and secondary bluff bodies,

8 respectively. $\rho_{\text {a }}$ represents the air density; $H_{i}$ and $U_{i}$ are the height of the bluff bodies and the wind

9 velocity applied to each bluff bodies. $C_{g i}$ represents the dimensionless coefficients of the aerodynamic

10 transverse force, which is a function of attack angle according to the assumption of quasi-steady state of

11 the bluff bodies in wind flow field referring to the previous studies $[19,37] . C_{g i}$ is the inherent

12 characteristics related to the cross-section shape of the bluff body and Renolds number, which can be

13 obtained through series expansion of the transverse aerodynamic force at different rotation angles of the

14 bluff body. It can be expressed as $C_{g i}\left(\alpha_{i}\right)=A_{1 i} \alpha_{i}+A_{3 i} \alpha_{i}^{3}+A_{5 i} \alpha_{i}^{5}+\mathrm{L}$, with $\alpha_{i}=\&_{i} / U_{i}$, where $A_{1 i}, A_{3 i}$,

$15 A_{5 i}$ are the aerodynamic coefficients. In this study, a third-order expansion is adopted to describe the

16 nonlinear dynamic behaviors resulted from the aerodynamic force.

17 Subsequently, we performed several manipulations using variational calculus to Eqs. (2), (3) and

18 (7), then substituted the resultants into Eq. (1) to gather the coefficients of $\delta y_{1}, \delta y_{2}, \delta \lambda_{1}(t)$ and $\delta \lambda_{2}(t)$. 
1 Furthermore, we conducted a normalization manipulation for a calculation convenience, thus a set of 2 governing equations can be obtained as

$$
\begin{aligned}
& +\omega_{1} y_{1}+2 \omega_{1} \zeta_{1} \& \tau_{1}\left(y_{1}-y_{2}\right)+\tau_{3}\left(y_{1}-y_{2}\right)^{3}-\Theta_{e 1} V_{e 1}-\theta_{1} \&=F_{\mathrm{g} 1}^{\prime}, \\
& +\omega_{2} y_{2}+2 \omega_{2} \zeta_{2} \&-\tau_{1}\left(y_{1}-y_{2}\right)-\tau_{3}\left(y_{1}-y_{2}\right)^{3}-\Theta_{e 2} V_{e 2}-\theta_{2}^{6} \&=F_{\mathrm{g} 2} \text {, }
\end{aligned}
$$

$$
V_{1}^{\&}+\eta_{1} V_{1}+\not 2 q \alpha=0
$$

$$
V_{2}^{\&}+\eta_{2} V_{2}+\not l_{2} \&=0 \text {, }
$$

7 where the definitions of the normalized parameters in Eqs. (9) are listed in Table 1. The set of governing

8 equations given in Eq. (9) indicates that the two primary and secondary oscillators are mechanically

9 coupled to each other through a nonlinear magnetic force. Additionally, the oscillating aerodynamic

10 wake force generated by the adjacent bluff body during oscillation is considered to avoid the loss of

11 generality of the mathematical modeling although it has a tiny disturbance to the dynamic behaviors of

12 bluff body compared to the magnetic force. It should be noted that the oscillating wake force acting to

13 the bluff bodies is not equal due to the unilaterality of the incoming wind flow. Accordingly, an order

14 selection considering the wake force scale should be conducted in finding analytical solution using 15 perturbation method. 
1 Table 1 Normalized parameters used in the governing equations, where $i=1,2$ denotes the primary and 2 secondary oscillators, respectively.

\begin{tabular}{lc}
\hline Definition & Parameters \\
\hline Undamped natural frequencies & $\omega_{i}=\sqrt{k_{i} / m_{i}}$ \\
Damping ratios & $\zeta_{i}=c_{i} /\left(2 \sqrt{m_{i} k_{i}}\right)$ \\
Mass normalized electromechanical coupling coefficients & $\Theta_{e i}=\theta_{e i} / m_{i}$ \\
Normalized wake coupling coefficients & $\theta_{i}=\theta_{i}^{w} / m_{i}$ \\
Normalized magnetic force coefficients for primary oscillator & $\tau_{1}=\mathrm{T}_{1} / m_{1}, \tau_{3}=\mathrm{T}_{3} / m_{1}$ \\
Normalized magnetic force coefficients for secondary oscillator & $\tau \ell_{1}=\mathrm{T}_{1} / m_{2}, \tau f_{3}=\mathrm{T}_{3} / m_{2}$ \\
Capacitance normalized electromechanical coupling coefficients & $\ell_{i}=\theta_{e i} / C_{p i}$ \\
Equivalent impedances & $\eta_{i}=1 / R_{L i} C_{p i}$ \\
\hline
\end{tabular}

\subsection{Analytical solutions based on method of multiple scale}

In an attempt to understand the nonlinear dynamic behaviors of the present system, especially the 6 internal resonance between the individual oscillator, the perturbation method of multiple scale is utilized to obtain the approximate analytical solutions for Eq. (9). The time dependence can be expanded into 8 two time scales in the form $T_{0}=t$ (fast time scale) and $T_{1}=\varepsilon t$ (slow time scale), respectively, where $\varepsilon$ is a scaling parameter. The new time derivatives with respect to $t$ can be expressed as:

$$
\frac{\mathrm{d}}{\mathrm{d} t}=D_{0}+\varepsilon D_{1}+O\left(\varepsilon^{2}\right), \frac{\mathrm{d}^{2}}{\mathrm{~d} t^{2}}=D_{0}^{2}+2 \varepsilon D_{0} D_{1}+O\left(\varepsilon^{2}\right)
$$

11 where $D_{n}=\frac{\partial}{\partial T_{n}}$ and $O\left(\varepsilon^{2}\right)$ denotes the terms higher than the second power of $\varepsilon$. The asymptotic 
series solutions are expanded up to the first order, such that

$$
\begin{aligned}
& y_{1}\left(T_{0}, T_{1}\right)=\varepsilon^{0}\left(y_{1}\right)_{0}\left(T_{0}, T_{1}\right)+\varepsilon\left(y_{1}\right)_{1}\left(T_{0}, T_{1}\right), \\
& y_{2}\left(T_{0}, T_{1}\right)=\varepsilon^{0}\left(y_{2}\right)_{0}\left(T_{0}, T_{1}\right)+\varepsilon\left(y_{2}\right)_{1}\left(T_{0}, T_{1}\right), \\
& v_{1}\left(T_{0}, T_{1}\right)=\varepsilon^{0}\left(v_{1}\right)_{0}\left(T_{0}, T_{1}\right)+\varepsilon\left(v_{1}\right)_{1}\left(T_{0}, T_{1}\right), \\
& v_{2}\left(T_{0}, T_{1}\right)=\varepsilon^{0}\left(v_{2}\right)_{0}\left(T_{0}, T_{1}\right)+\varepsilon\left(v_{2}\right)_{1}\left(T_{0}, T_{1}\right),
\end{aligned}
$$

3 where the symbols of ()$_{0}$ and ()$_{1}$ represent the $\varepsilon^{0}$ and $\varepsilon^{1}$ order solution for the dynamic responses

4 of the primary and secondary oscillators. The oscillators in the present system are assumed to be weakly

5 coupled by the external magnetic force and oscillating wake force. An ordering for the terms in Eq. (9)

6 can be determined by scaling the viscous damping, magnetic coupling, electromechanical coupling, and

7 aerodynamic force in the same order. In other words, the scaled relevant coefficients or terms can be

8 expressed as

$$
\begin{aligned}
& \zeta_{1}=\varepsilon \zeta_{1}, \zeta_{2}=\varepsilon \zeta_{2}, \tau_{1}=\varepsilon \tau_{1}, \tau_{3}=\varepsilon \tau_{3}, \tau \tau_{1}=\varepsilon t_{1},
\end{aligned}
$$

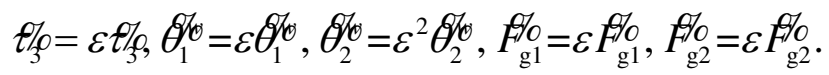

10 It should be noted that the oscillating wake coupling coefficient generated by the secondary oscillator is

11 defined to higher order of $\varepsilon^{2}$ considering the fact that the effect of the wake generated from the

12 secondary oscillator to the primary bluff body is much weaker than that from the primary oscillator to

13 the secondary oscillator generated. Substituting the scaled terms into Eq. (10) and expanding the

14 governing equations in terms of Eq. (10) and (12), we can obtain the ordered equations after collecting

15 the coefficients of $\varepsilon^{0}$ and $\varepsilon^{1}$, which yields the following results:

$16 \varepsilon^{0}$ order equations: 


$$
\frac{\partial^{2}}{\partial T_{0}^{2}}\left(y_{1}\right)_{0}+\omega_{1}^{2}\left(y_{1}\right)_{0}=0
$$

$$
\frac{\partial^{2}}{\partial T_{0}^{2}}\left(y_{2}\right)_{0}+\omega_{2}^{2}\left(y_{2}\right)_{0}=0
$$

$$
c_{p} \frac{\partial}{\partial T_{0}}\left(v_{1}\right)_{0}+\theta_{e} \frac{\partial}{\partial T_{0}}\left(y_{1}\right)_{0}+\frac{1}{R_{L 1}}\left(v_{1}\right)_{0}=0,
$$

4

$$
c_{p} \frac{\partial}{\partial T_{0}}\left(v_{2}\right)_{0}+c_{p} \theta_{e} \frac{\partial}{\partial T_{0}}\left(y_{2}\right)_{0}+\frac{1}{R_{L 2}}\left(v_{2}\right)_{0}=0 .
$$

$5 \quad \varepsilon^{1}$ order equations:

6

$$
\begin{aligned}
& \frac{\partial^{2}}{\partial T_{0}^{2}}\left(y_{1}\right)_{1}+\omega_{1}^{2}\left(y_{1}\right)_{1}=-2 \zeta_{1} \omega_{1} \frac{\partial}{\partial T_{0}}\left(y_{1}\right)_{0}+3 \tau_{3}\left(y_{1}\right)_{0}{ }^{2}\left(y_{2}\right)_{0}-3 \tau_{3}\left(y_{1}\right)_{0}\left(y_{2}\right)_{0}^{2}-2 \frac{\partial^{2}}{\partial T_{0} \partial T_{1}}\left(y_{1}\right)_{0}, \\
& +n_{1} U_{1} \mathrm{~A}_{11} \frac{\partial}{\partial T_{0}}\left(y_{1}\right)_{0}+\frac{n_{1} \mathrm{~A}_{13}}{U_{1}}\left(\frac{\partial}{\partial T_{0}}\left(y_{1}\right)_{0}\right)^{3}-\tau_{1}\left(y_{1}\right)_{0}+\tau_{1}\left(y_{2}\right)_{0}-\tau_{3}\left(y_{1}\right)_{0}^{3}+\tau_{3}\left(y_{2}\right)_{0}^{3}+\chi_{1}\left(v_{1}\right)_{0},
\end{aligned}
$$

7

$$
\begin{aligned}
& \frac{\partial^{2}}{\partial T_{0}^{2}}\left(y_{2}\right)_{1}+\omega_{2}^{2}\left(y_{2}\right)_{1}=-2 \zeta_{2} \omega_{2} \frac{\partial}{\partial T_{0}}\left(y_{2}\right)_{0}-3 \operatorname{tg}\left(y_{1}\right)_{0}{ }^{2}\left(y_{2}\right)_{0}+3 \operatorname{tg}\left(y_{1}\right)_{0}\left(y_{2}\right)_{0}{ }^{2}-2 \frac{\partial^{2}}{\partial T_{0} \partial T_{1}}\left(y_{2}\right)_{0}
\end{aligned}
$$

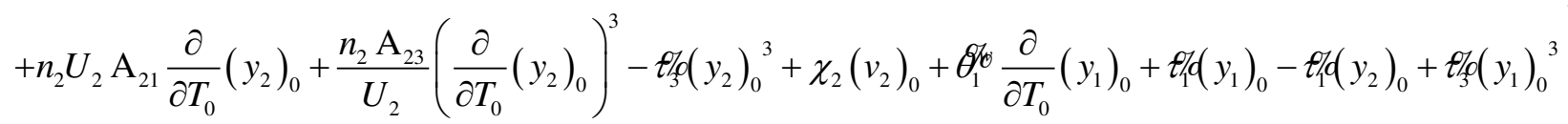

8

$$
\begin{gathered}
c_{p} \frac{\partial}{\partial T_{0}}\left(v_{1}\right)_{1}+\theta_{e} \frac{\partial}{\partial T_{0}}\left(y_{1}\right)_{1}+\frac{1}{R_{L 1}}\left(v_{1}\right)_{1}=-c_{p} \frac{\partial}{\partial T_{1}}\left(v_{1}\right)_{0}-\theta_{e} \frac{\partial}{\partial T_{1}}\left(y_{1}\right)_{0}, \\
c_{p} \frac{\partial}{\partial T_{0}}\left(v_{2}\right)_{1}+c_{p} \theta_{e} \frac{\partial}{\partial T_{0}}\left(y_{2}\right)_{1}+\frac{1}{R_{L 2}}\left(v_{2}\right)_{1}=-c_{p} \frac{\partial}{\partial T_{1}}\left(v_{2}\right)_{0}-c_{p} \theta_{e} \frac{\partial}{\partial T_{1}}\left(y_{2}\right)_{0} .
\end{gathered}
$$

10 The solutions of the zeroth-order Eqs. (13)-(16) can be written as:

11

$$
\left(y_{1}\right)_{0}=A_{1}\left(T_{1}\right) \mathrm{e}^{i \omega_{1} T_{0}}+c c
$$




$$
\left(y_{2}\right)_{0}=B_{1}\left(T_{1}\right) \mathrm{e}^{i \omega_{2} T_{0}}+c c
$$

$$
\left(v_{1}\right)_{1}=-\frac{i \theta_{e} \omega_{1} A_{1}\left(T_{1}\right) R_{L 1} \mathrm{e}^{\omega_{1} T_{0} i}}{i R_{L 1} c_{p} \omega_{1}+1}+\frac{i \theta_{e} \omega_{1} \overline{A_{1}}\left(T_{1}\right) R_{L 1}}{\left(1-i R_{L 1} c_{p} \omega_{1}\right) \mathrm{e}^{\omega_{1} T_{0} i}}+E_{1}\left(T_{1}\right) \mathrm{e}^{-\frac{T_{0}}{R_{L 1} c_{p}}},
$$

$$
\left(v_{2}\right)_{1}=-\frac{i \theta_{e} \omega_{2} B_{1}\left(T_{1}\right) R_{L 2} \mathrm{e}^{\omega_{2} T_{0} i}}{i R_{L 2} c_{p} \omega_{2}+1}+\frac{i \theta_{e} \omega_{2} \overline{B_{1}}\left(T_{1}\right) R_{L 2}}{\left(1-i R_{L 2} c_{p} \omega_{2}\right) \mathrm{e}^{\omega_{2} T_{0} i}}+E_{2}\left(T_{1}\right) \mathrm{e}^{-\frac{T_{0}}{R_{L 2} c_{p}}}
$$

4 where cc denotes the complex conjugate of the preceding term. $A_{1}$ and $B_{1}$ denote the unknown complex-valued functions for primary and secondary oscillators, respectively. The over-bar denotes the complex conjugate. Substituting the zeroth-order solutions into the right hand side of the first order equations (17-20) and expanding the equations, it can be observed that frequency modes with frequency

8 ratio of 1:1 are coupled via the oscillating wake force and the linear terms of magnetic force.

9 Additionally, it also can be observed that the frequency modes with frequency ratio of 1:3 are coupled

10 via cubic nonlinearities of the magnetic force. In this study, the 1:1 internal resonance plays a decisive

11 role in the improvement of dynamic behaviors and the output performance for the present system

12 referring to the results of a preliminary numerical analysis. After eliminating the undesired non-secular

13 terms and collecting coefficients of harmonics, it can be obtained as follows:

$$
\begin{gathered}
\frac{\partial^{2}}{\partial T_{0}^{2}}\left(y_{1}\right)_{1}+\omega_{1}^{2}\left(y_{1}\right)_{1}=Q_{1} e^{i \omega_{1} T_{0}}+Q_{2} e^{i \omega_{2} T_{0}}+c c+N S T, \text { with } \\
Q_{1}=\frac{3 i n_{1} A_{13} A_{1}\left(T_{1}\right)^{2} \omega_{1}^{3} \bar{A}_{1}\left(T_{1}\right)}{U_{1}}-\frac{i \Theta_{e 1} \theta_{e} \omega_{1} A_{1}\left(T_{1}\right) R_{L 1}}{i R_{L 1} c_{p} \omega_{1}+1}-\tau_{1} A_{1}\left(T_{1}\right)-3 \tau_{3} \bar{A}_{1}\left(T_{1}\right) A_{1}\left(T_{1}\right)^{2}, \\
-2 i\left(\frac{\mathrm{d}}{\mathrm{dT}} A_{1}\left(T_{1}\right)\right) \omega_{1}+i n_{1} U_{1} A_{11} A_{1}\left(T_{1}\right) \omega_{1}-6 \tau_{3} A_{1}\left(T_{1}\right) \bar{B}_{1}\left(T_{1}\right) B_{1}\left(T_{1}\right)-2 i \zeta_{1} \omega_{1}^{2} A_{1}\left(T_{1}\right) \\
Q_{2}=6 \tau_{3} \overline{A_{1}}\left(T_{1}\right) A_{1}\left(T_{1}\right) B_{1}\left(T_{1}\right)+3 \tau_{3} \bar{B}_{1}\left(T_{1}\right) B_{1}\left(T_{1}\right)^{2}+\tau_{1} B_{1}\left(T_{1}\right) .
\end{gathered}
$$




$$
\frac{\partial^{2}}{\partial T_{0}^{2}}\left(y_{2}\right)_{1}+\omega_{2}^{2}\left(y_{2}\right)_{1}=Q_{3} \mathrm{e}^{\omega_{1} T_{0} i}+Q_{4} \mathrm{e}^{i \omega_{2} T_{0}}+c c+N S T, \text { with }
$$

$$
Q_{3}=3 \tau_{3} \overline{A_{1}}\left(T_{1}\right)\left(A_{1}\left(T_{1}\right)\right)^{2}+6 \tau_{3} A_{1}\left(T_{1}\right) \overline{B_{1}}\left(T_{1}\right) B_{1}\left(T_{1}\right)+i \Theta_{w} A_{1}\left(T_{1}\right) \omega_{1}+\tau_{1} A_{1}\left(T_{1}\right)
$$

$$
Q_{4}=3 \frac{i n_{2} A_{23}\left(B_{1}\left(T_{1}\right)\right)^{2} \omega_{2}^{3} \overline{B_{1}}\left(T_{1}\right)}{U_{2}}-62 \% \overline{A_{1}}\left(T_{1}\right) A_{1}\left(T_{1}\right) B_{1}\left(T_{1}\right)-2 i \zeta_{2} \omega_{2}{ }^{2} B_{1}\left(T_{1}\right)-2 i\left(\frac{\mathrm{d}}{\mathrm{d} T_{1}} B_{1}\left(T_{1}\right)\right) \omega_{2} \text {. }
$$

$$
-3 \epsilon \bar{g}_{\overline{3}}\left(T_{1}\right)\left(B_{1}\left(T_{1}\right)\right)^{2}-\frac{i \Theta_{e 2} \theta_{e} \omega_{2} B_{1}\left(T_{1}\right) c_{p} R_{L 2}}{i R_{L 2} c_{p} \omega_{2}+1}+i n_{2} U_{2} A_{21} B_{1}\left(T_{1}\right) \omega_{2}-\tau_{1} B_{1}\left(T_{1}\right)
$$

5 where NST stands for the terms that do not produce secular terms. Then, it is necessary to introduce the $1: 1$ internal resonance condition as

$$
\omega_{2}=\sigma \varepsilon+\omega_{1}, \omega_{1}=-\sigma \varepsilon+\omega_{2}
$$

where $\sigma$ is the internal resonance detuning parameter, which represents the closeness of the two natural (26) and after a mathematical manipulation, the simplified equations can be expressed as

$$
\frac{\partial^{2}}{\partial T_{0}^{2}}\left(y_{1}\right)_{1}+\omega_{1}^{2}\left(y_{1}\right)_{1}=\left(Q_{1}+Q_{2} e^{i \sigma T_{1}}\right) e^{i \omega_{1} T_{0}}+c c+N S T
$$

$$
\frac{\partial^{2}}{\partial T_{0}^{2}}\left(y_{2}\right)_{1}+\omega_{2}^{2}\left(y_{2}\right)_{1}=\left(Q_{3}+Q_{4} e^{-i \sigma T_{1}}\right) e^{i \omega_{2} T_{0}}+c c+N S T
$$

13 Eliminating the terms that lead to secular terms in the above equations, and expressing the complex-

14 valued function in the polar form 


$$
A_{1}\left(T_{1}\right)=\frac{a\left(T_{1}\right) \mathrm{e}^{i \beta_{1}\left(T_{1}\right)}}{2}, B_{1}\left(T_{1}\right)=\frac{b\left(T_{1}\right) \mathrm{e}^{i \beta_{2}\left(T_{1}\right)}}{2}
$$

2 where $a$ and $b$ represent the unknown amplitude for the primary and secondary oscillators; $\beta_{1}$ and $\beta_{2}$

3 represent the phase of the corresponding dynamic responses. Next, separating the real and imaginary

4 parts in Eqs. (28)-(29) yields the modulation equations as

$5 \frac{\mathrm{d} a}{\mathrm{~d} T_{1}}=\frac{U_{1} b \sin (\Upsilon)\left(6 \tau_{3} a^{2}+3 \tau_{3} b^{2}+4 \tau_{1}\right)+a\left(4 U_{1}^{2} n_{1} \mathrm{~A}_{11} \omega_{1}-8 U_{1} \omega_{1}^{2} \zeta_{1}-4 U_{1} E_{12}\right)+3 a^{3} n_{1} \mathrm{~A}_{13} \omega_{1}^{3}}{8 U_{1} \omega_{1}}$,

$6 \quad \frac{\mathrm{d} \beta_{1}}{\mathrm{~d} T_{1}}=\frac{a\left(4 E_{11}+3 \tau_{3} a^{2}+6 \tau_{3} b^{2}+4 \tau_{1}\right)-\left(6 \tau_{3} a^{2} b+3 \tau_{3} b^{3}+4 \tau_{1} b\right) \cos (\Upsilon)}{8 \omega_{1} a}$,

$7 \quad \frac{\mathrm{d} b}{\mathrm{~d} T_{1}}=\frac{\left.-a U_{2} \sin (\Upsilon)(3 t){ }_{3}^{2}+6 t_{3}^{2} b^{2}+4 \tau_{1}\right)+4 U_{2} a \omega_{1} \Theta_{w} \cos (\Upsilon)+3 b^{3} \omega_{2}^{3} n_{2} \mathrm{~A}_{23}+\left(4 \omega_{2} U_{2} n_{2} \mathrm{~A}_{21}-8 \zeta_{2} \omega_{2}^{2}-4 E_{22}\right) U_{2} b}{8 \omega_{2} U_{2}}$,

$8 \quad \frac{\mathrm{d} \beta_{2}}{\mathrm{~d} T_{1}}=\frac{\left(-3 t / a_{3} a^{3}-6 t / 3 a b^{2}-4 t f_{1} a\right) \cos (\Upsilon)-4 a \omega_{1} \Theta_{w} \sin (\Upsilon)-b\left(-6 t / 3 a^{2}-3 t / b^{2} b^{2}+4 E_{21}-4 t \tau_{1}\right)}{8 \omega_{2} b}$, with

$$
\begin{gathered}
\gamma=\sigma T_{1}+\beta_{2}-\beta_{1}, E_{11}=\frac{\Theta_{e 1} \theta_{e} c_{p} \omega_{1}^{2} R_{L 1}^{2}}{R_{L 1}{ }^{2} c_{p}{ }^{2} \omega_{1}^{2}+1} E_{12}=\frac{\Theta_{e 1} \theta_{e} \omega_{1} R_{L 1}}{R_{L 1}{ }^{2} c_{p}{ }^{2} \omega_{1}{ }^{2}+1}, \\
E_{21}=\frac{\Theta_{e 2} \theta_{e} c_{p} \omega_{2}{ }^{2} R_{L 2}^{2}}{R_{L 2}{ }^{2} c_{p}{ }^{2} \omega_{2}{ }^{2}+1} E_{22}=\frac{\Theta_{e 2} \theta_{e} \omega_{2} R_{L 2}}{R_{L 2}{ }^{2} c_{p}{ }^{2} \omega_{2}{ }^{2}+1} .
\end{gathered}
$$

11 The time derivatives of $\beta_{1}$ and $\beta_{2}$ in Eq. (32) and (34) can be vanished in terms of the relationship of $12 \gamma^{\prime}=\sigma+\beta_{2}^{\prime}-\beta_{1}^{\prime}$, where the primer means the time derivation of $T_{1}$, and thus the modulation equations

13 with 4-dimensional phase space $\left(a, b, \beta_{1}, \beta_{2}\right)$ can be reduced to a 3-dimensional phase space $(a, b, \gamma)$.

14 Additionally, the steady-state responses can be determined by setting the time derivatives in the 
1 modulation equations equal to zero. The resulted equations are a set of transcendental equations which

2 are difficult to be solved by an analytical method, however, it is fairly straightforward to be solved

3 numerically for semi-analytical solutions. Subsequently, we can obtain the output voltage generated by

4 the piezoelectric element using Eqs. (23) and (24), where homogeneous and non-homogeneous solutions

5 are contained in them. It is known that the homogeneous solutions would converge to zero on account of

6 the electric energy dissipation in the external resistor along the time. Consequently, we can find the

7 output voltage amplitude through the non-homogeneous terms, and the resultants can be expressed with

8 a function of the amplitude of the corresponding oscillators as

$$
V_{1}=\frac{\theta_{e} \omega_{1} R_{L 1}}{\sqrt{R_{L 1}^{2} c_{p}^{2} \omega_{1}^{2}+1}}|a|, V_{2}=\frac{\theta_{e} \omega_{2} R_{L 2}}{\sqrt{R_{L 2}^{2} c_{p}^{2} \omega_{2}^{2}+1}}|b|
$$

10 Subsequently, the output power can be described as

$$
P_{1}=\frac{\theta_{e}^{2} \omega_{1}^{2} R_{L 1}}{R_{L 1}^{2} c_{p}^{2} \omega_{1}^{2}+1}|a|^{2}, P_{2}=\frac{\theta_{e}^{2} \omega_{2}^{2} R_{L 2}}{R_{L 2}^{2} c_{p}^{2} \omega_{2}^{2}+1}|b|^{2}
$$

12 According to the expressions of output voltage and power, we can compare the energy harvesting

13 performance between the internal resonance case and non-internal resonance case. 
3 Oscillating wake induced internal resonance

2 Table 2 Physical parameters used in the proposed system.

\begin{tabular}{lcc}
\hline Description & Symbol & Value \\
\hline Mass of bluff bodies & $m_{1}, m_{2}$ & $0.015 \mathrm{~kg}, 0.01 \mathrm{~kg}$ \\
Characteristic dimension of the bluff bodies & $D$ & $30 \mathrm{~mm}$ \\
Height of the bluff bodies & $H_{1}, H_{2}$ & $100 \mathrm{~mm}, 80 \mathrm{~mm}$ \\
Damping constants & $c_{1}, c_{2}$ & $0.0021 \mathrm{Ns} / \mathrm{m}, 0.0015 \mathrm{Ns} / \mathrm{m}$ \\
Stiffness of spring & $k_{1}$ & $236.86 \mathrm{~N} / \mathrm{m}$ \\
Electromechanical coupling coefficients & $\theta_{e 1}=\theta_{e 2}=\theta_{e}$ & $1.1 \times 10^{-4} \mathrm{~N} / \mathrm{V}$ \\
Capacitance of the piezoelectric patches & $C_{p 1}=C_{p 2}=C_{p}$ & $90 \mathrm{nF}$ \\
$1^{\text {st }}$ and ${ }^{\text {rd }}$ order aerodynamic coefficients [38] & $A_{11}=A_{21}, A_{13}=A_{23}$ & $2.3,-18.4$ \\
Air density & $\rho_{a}$ & $1.225 \mathrm{~kg} / \mathrm{m}^{3}$ \\
Magnitudes of magnetization & $\bar{m}_{1}=\bar{m}_{2}$ & $0.295 \times 10^{6} \mathrm{~A} / \mathrm{m}$ \\
Volumes of magnets & $\bar{v}_{1}=\bar{v}_{2}$ & $40 \pi \mathrm{mm}$ \\
Vacuum permeability & $\mu_{0}$ & $4 \pi \times 10^{-7} \mathrm{H} / \mathrm{m}^{3}$ \\
\hline
\end{tabular}

In this section, we first investigated the electric characteristics of the external circuit and some

5 fundamental aerodynamic features as a preliminary investigation for the further examination of coupled

6 dynamic behaviors. Additionally, the oscillating wake generated from the primary bluff body has an

7 effect to the dynamic behaviors secondary bluff body. To validate the proposed analytical model and

8 give a comprehensive understanding of oscillating wake coupled system, we examine the dynamic

9 behaviors considering the pure oscillating wake coupling effect by assuming the magnet gap distance to

10 be infinite. Subsequently, some bifurcation analyses are carried out to clarify the inherent characteristics 
1 of the internal resonance. The relevant parameters and the corresponding values used in this study are

$2 \quad$ listed in Table 2.

\subsection{Effect of the external resistance}

In a wind energy harvesting system, the critical wind speed is one of the most important factors to

5 evaluate the output performance. Here, we first investigate and clarify the influence of external load to

6 the critical wind speed in terms of the secular equations. The energy harvesting system would loss

7 stability when the linear mechanical damping changes to be negative. Nevertheless, the resultant

8 damping term is determined by the combined effect of the mechanical damping and the electric damping

9 in an electromechanical coupled system. Based on the simplified secular equations, the derived damping

10 coefficient can be expressed as

$$
U_{i} \mathrm{~A}_{21} \omega_{i} n_{i}-2 \zeta_{i} \omega_{i}^{2}-E_{i 2} \geq 0
$$

12 The critical wind speed can thus be expressed as

$$
U_{c r i}=\frac{2 \zeta_{i} \omega_{i}+\frac{\Theta_{e i} \theta_{e} R_{L i}}{R_{L i}^{2} c_{p}^{2} \omega_{i}^{2}+1}}{n_{i} A_{11}} .
$$
external resistance and the critical wind speed. As shown in Fig. 2, the critical wind speed changes to be higher as the external resistance increases and then decline with the further increase of the external

17 resistance, which matches the results in [39]. The up and down phenomenon of critical wind speed 
versus external resistance is resulted from the electromechanical damping terms of $E_{i 2}$, which causes an

2 maximum value damping at the resistance value of $0.176 \mathrm{M} \Omega$ for the primary oscillator. The resistance

3 value is also regarded as an optimal one to obtain a maximum output power in the external circuit. As

4 shown in Fig. 2(a), the electromechanical coupling strength also shows a significant influence to the

5 critical wind speed. It can be observed that the strong coupling effect between electric domain and

6 mechanical domain would result in an increase of critical wind speed. On the other hand, the detuning

7 parameter $\sigma$ describes the difference between the natural frequencies of the primary and secondary

8 oscillators, which causes the change of the secondary natural frequency as shown in Fig. 2(b). It further

9 indicates the fact that the increase of natural frequency is able to pull down the critical wind speed.

10 In a general energy harvesting system, impedance matching between mechanical and electric

11 system is essential to obtain a maximum output power. Mechanical energy would be dissipated during

12 energy harvesting operation due to the fact that the mechanical energy is transformed into electric energy.

13 However, the increase of electric damping results in a dramatic decrease in the dynamic response of

14 oscillator as shown in Fig. 3, especially in the low wind speed range. This result gives a pertinent

15 interpretation for the fact that the critical wind speed is postponed as the external resistance near to the

16 optimal value as shown in Fig. 2. For a consistent evaluation of the effect of internal resonance and other

17 key parameters, the external resistance is treated to be infinite, in other words, an open circuit in the next

18 section. 

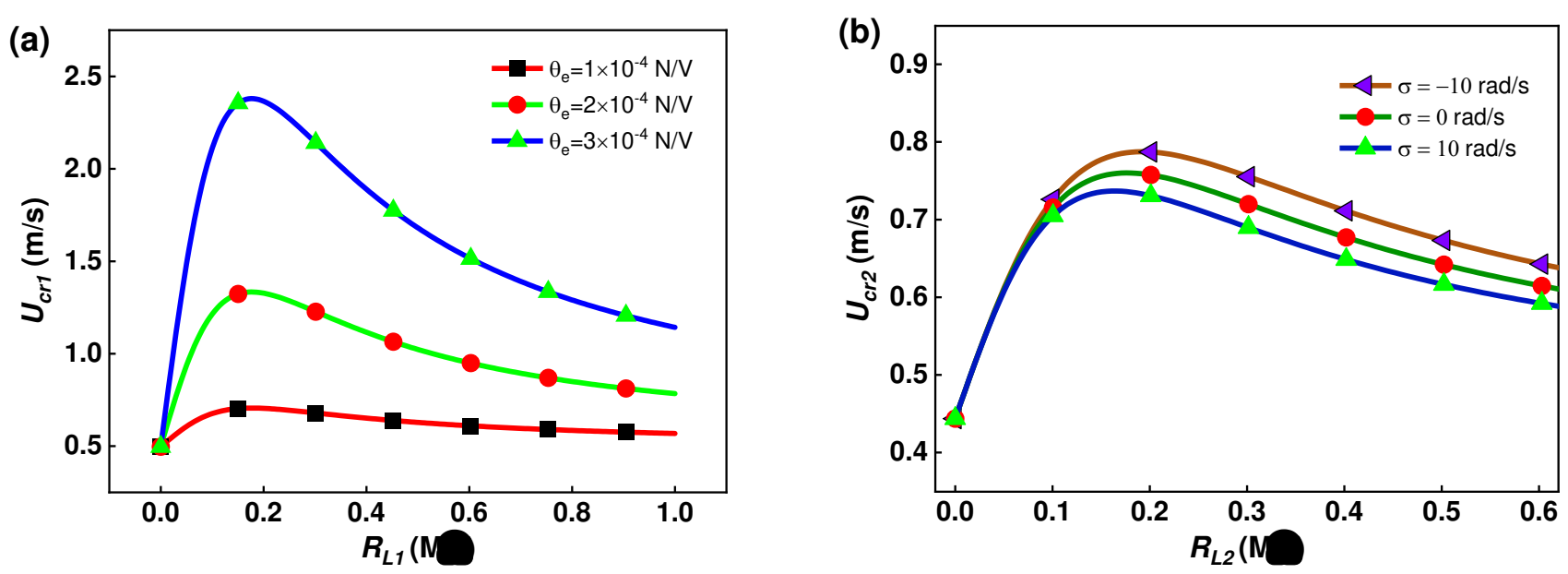

Fig. 2 Variation of critical wind speed versus external resistance with different (a) electromechanical coupling coefficients, (b) detuning parameters.

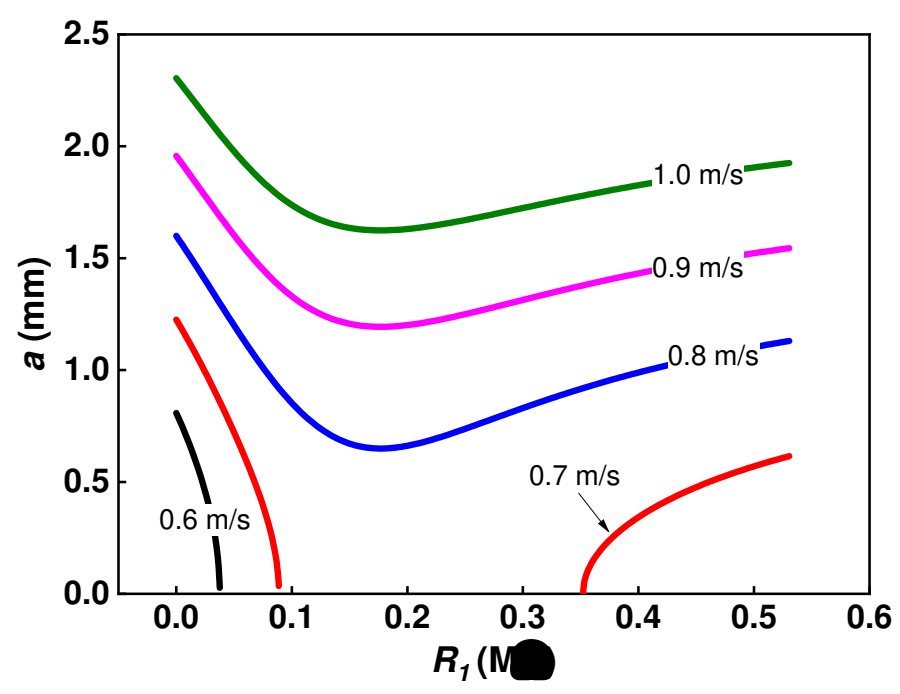

6 Fig. 3 Electric damping effects to the amplitude of the primary oscillator under different wind speeds.

\subsection{Parametric analyses under wake effect}

In this subsection, we investigated the dynamic responses of both oscillators under no magnetic

9 force coupling by setting the distance between two magnets infinity. The oscillating wake generated by 
1 the primary bluff body periodically acts on the secondary bluff body, however, it is not easy to inversely

2 affect that of the primary bluff body. This phenomenon is analytically and numerically investigated, and

3 the results are shown in Fig. 4(a). In this numerical calculation, we adopted a means of wind speed

4 sweeping to illustrate the evolution of dynamic behavior with time. Through comparing the numerical

5 results to the analytical ones, a good matching can be seen in a wind speed range from $0 \mathrm{~m} / \mathrm{s}$ to $10 \mathrm{~m} / \mathrm{s}$.

6 The strength of the incoming wind exerted on the secondary bluff body tends to be weaker due to

7 the wind flow obstruction of the primary bluff body configured in front of the secondary bluff body, in

8 other words, the actual wind speed loaded to the secondary bluff body is smaller than that to the primary

9 one. This fact necessitates a function relationship between the wind speeds of $U_{1}$ and $U_{2}$. We assume

10 that $U_{2}$ is linearly related to $U_{1}$ and expressed as $U_{2}=\eta U_{1}$. Here, $\eta$ is a wind speed proportional

11 constant to quantitatively express the reduction of wind speed, which always smaller than one.

12 Subsequently, we analytically investigated the amplitude responses of the secondary bluff body versus

13 wind speed under different $\eta$ with $\Theta_{w}=0 \mathrm{Ns} / \mathrm{m}$ and $\sigma=0 \mathrm{rad} / \mathrm{s}$ as shown in Fig.4 (b). It can be seen that

14 the amplitudes of the bluff body increase along the axis of the wind speed for all the various $\eta$ cases.

15 However, the critical wind speed moves to the higher range as $\eta$ decreases. The subplot in Fig.4 (b)

16 shows the variation of dimensionless critical wind speed $\bar{U}_{c r}\left(U_{c r} / U_{0}\right)$ over the wind speed

17 proportional constant $\eta$, where $U_{0}$ denotes the critical wind speed when $\eta$ is zero. It further shows that

$18 \bar{U}_{c r}$ is much sensitive to the variation of $\eta$ at low range of $\eta$, which demonstrated a dramatical increase

19 as $\eta$ close to zero. 

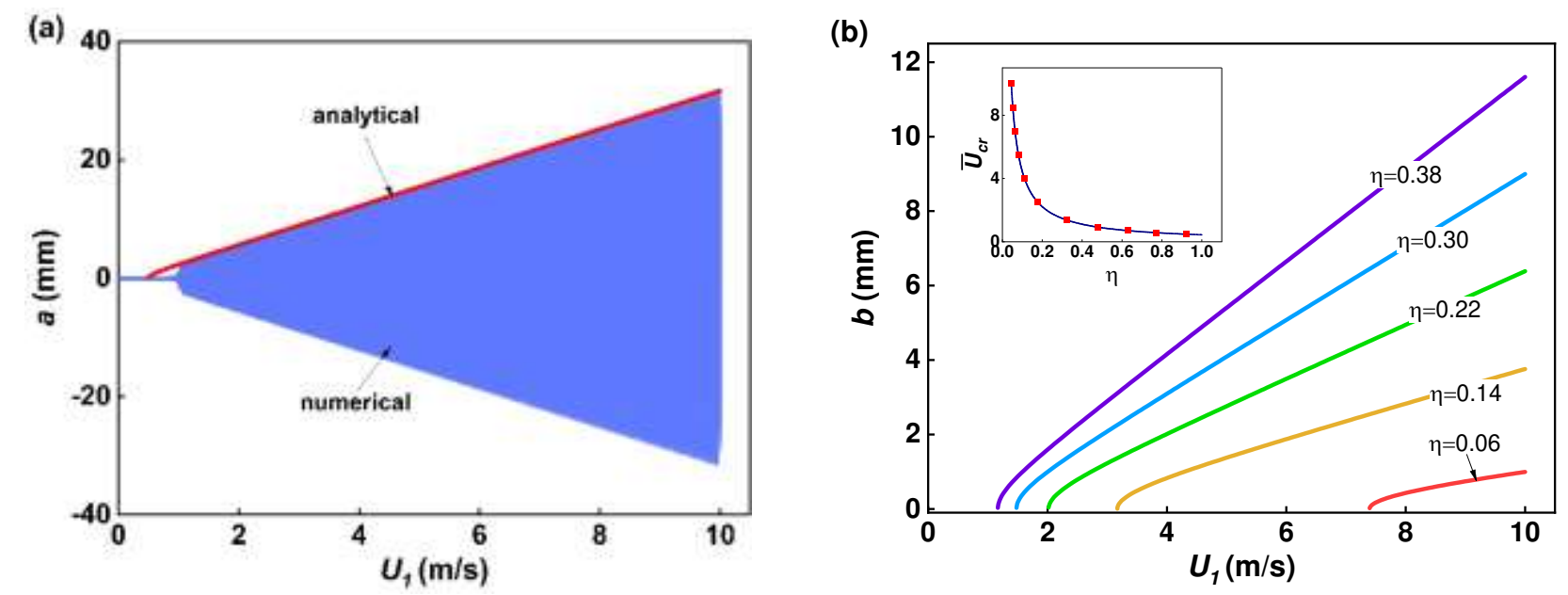

2 Fig. 4 (a) Results comparisons Electric damping effects to the amplitude of the primary oscillator under different wind speeds with $\Theta_{w}=0 \mathrm{Ns} / \mathrm{m}$ and $\sigma=0 \mathrm{rad} / \mathrm{s}$.

A natural difference of the dynamic responses happens in the secondary bluff body when the wake coupling effect is accounted for in the dynamical analysis. Response bifurcation and multiple solution

6 phenomenon start to appear when $\Theta_{w}$ is given a small value of 0.002 Ns/m as shown in Fig. 5. Here,

7 two stable branches and one unstable branch can be determined through the stability evaluation based on

8 the eigenvalues of the Jacobian matrix of the right-hand sides of Eqs. (31) - (34) as stated in [40]. The

9 cross point between the bifurcation curve and the dynamic response curve is defined as the saddle node.

10 It can be clearly observed that the critical wind speed is pulled down to the lower wind range referring to

11 that case without consideration of wake oscillation. In addition, it is also observed that the saddle node

12 can be significantly reduced from $9.2 \mathrm{~m} / \mathrm{s}$ to $3.8 \mathrm{~m} / \mathrm{s}$ as $\eta$ increases from 0.08 to 0.16 , and the second

13 stable branch tends to be close to the first stable branch as $\eta$ increases as illustrated in Fig. 5 (a) and (b). 

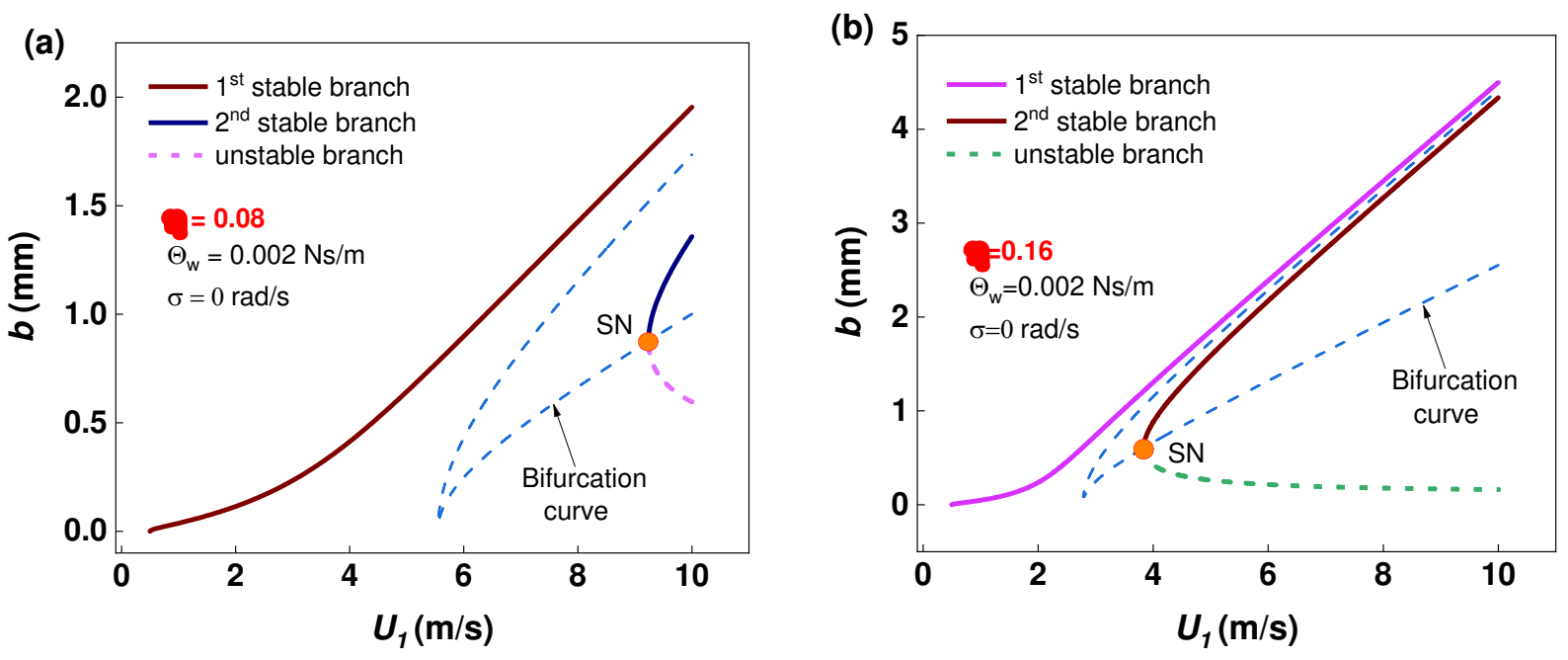

2 Fig. 5 Bifurcation graph of secondary bluff body response under (a) $\eta=0.08$ and (b) $\eta=0.16$ with $\Theta_{w}$ $=0.020 \mathrm{Ns} / \mathrm{m}$ and $\sigma=0 \mathrm{rad} / \mathrm{s}$.
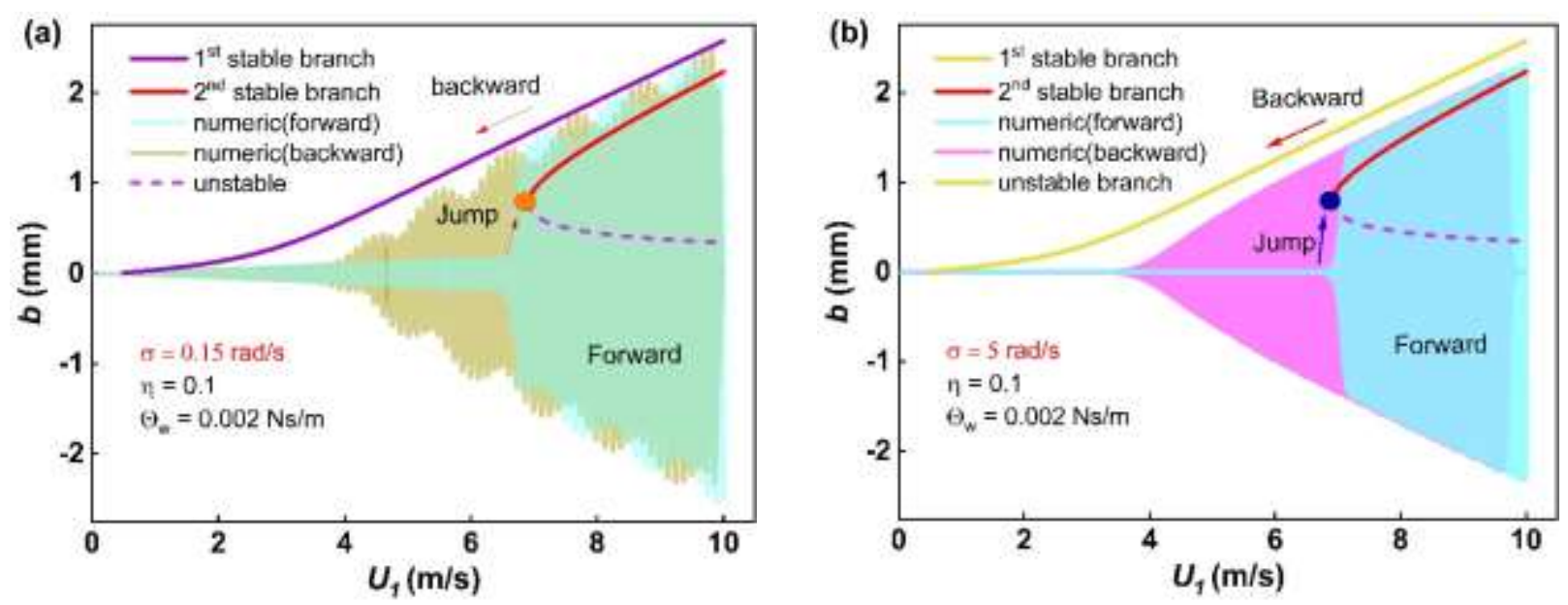

Fig. 6 comparisons between analytical prediction results and numerical results with forward and backward sweeping of wind speed under (a) $\sigma=0.15 \mathrm{rad} / \mathrm{s}$ and (b) $\sigma=5 \mathrm{rad} / \mathrm{s}$ with $\Theta_{w}=0.020 \mathrm{Ns} / \mathrm{m}$ and $\eta=0.1$

The influence of detuning parameter $\sigma$ to the dynamic response of the secondary bluff body is also investigated in the presence of oscillating wake coupling as shown in Fig. 6. The graphs depicted similar 
1 few effects to the saddle node position, which is much different with the varying case of $\eta$. To further evaluate the analytical results, a series of numerical simulation have been conducted by sweeping the parameter with variation of time. A jump phenomenon occurs at the saddle node point when the wind speed increases from zero to higher wind speed range both in $\sigma=0.15$ and $\sigma=5$ cases as shown in Fig.

6 (a) and (b). It is worth noting that the maximum amplitude appears close to the first stable branch at a smaller $\sigma$ and which tends to approach the second stable branch when a bigger $\sigma$ is introduced. On the other hand, the amplitude responses decrease along the routine of the first stable branch when a wind speed decreases backward. From the above comparisons, a good matching between the analytical and numerical results are achieved, which further validates the proposed prediction model.

\subsection{Perspective in saddle-node bifurcation}

11 As previously mentioned, the saddle node point appears as we vary the associated parameters, the saddle node plays an important role in the dynamic response evaluation for a nonlinear coupled system.

13 Accordingly, a systematical analysis for the overall bifurcation curve is necessary at the viewpoint of

14 revealing the mechanism contained in the wake coupled oscillators. Response bifurcation usually

15 happens at the positions where the derivative of the response to the varying parameter is zero. A 3-

16 Dimensional graph for the amplitude of the secondary oscillator in terms of two varying parameters can

17 be identified based on the resultant polynomial equation. It is expected that a surface fold phenomenon

18 of the dynamic response would happen due to the non-linearity of the coupled parameter and 19 aerodynamic force. 
(a)

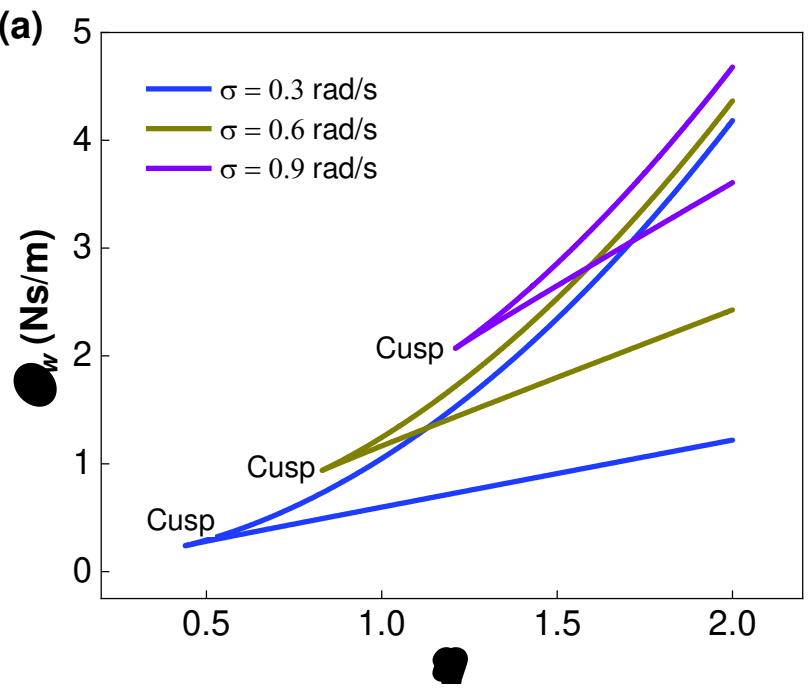

(b)

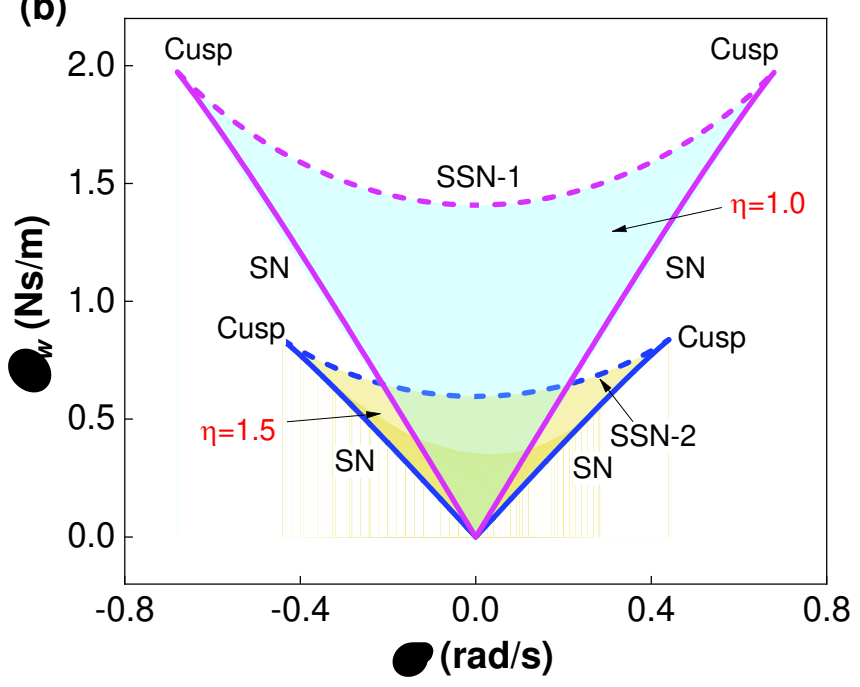

2 Fig. 7 Saddle node bifurcation of oscillating wake coupled nonlinear oscillator in (a) $\eta-\Theta_{w}$ plane and

3

For a coherent exhibition of the saddle-node bifurcation curve, we reduce the polynomial equation including 3 varying parameters to that including 2 parameters by vanishing the amplitude of secondary oscillator using the original polynomial equation and its derivative with the amplitude of secondary oscillator. As shown in Fig. 7, a series of saddle-node bifurcation curves are generated in $\eta-\Theta_{w}$ and $\sigma-\Theta_{w}$ plane where the stable and unstable fixed points collide. Additionally, a cusp bifurcation occurs when two saddle-node bifurcation curves meet as shown in Fig. 7(a) and (b). The folds degenerate at these cusp point, that is, the frequency synchronization tends to be difficult when the varying parameter passing the cusp point. It shows that cusp point moves to higher values of $\eta$ and $\Theta_{w}$ as $\sigma$ increases as illustrated in Fig. 7(a). In Fig. 7(b), the shadow regions are bounded by the saddle node curves and saddle-node bifurcation curve for a saddle and a twice saddle cycles (SSN). There cusp points appear in each bounded region. We can see from this graph that the shadow region is symmetric with the axis of 
$1 \sigma=0$, which indicates the bounded synchronization region is strongly dependent on the natural

2 frequency difference of the two oscillators. Furthermore, $\eta$ gives a significant influence to the area of 3 the bounded regime, namely the increasing of $\eta$ causes area expansion of the bounded regime.

\section{Magnetically force induced internal resonance}

The fundamental dynamic behaviors of the oscillators undergoing oscillating wake coupling have

7 been parametrically investigated in the previous section without consideration of real physical

8 conditions. In galloping-based energy harvesting, an asymmetric bluff body is usually adopted to

9 generate the transverse aerodynamic force, which leads to vibration of oscillator as the system loses its

10 stability. In the galloping phenomenon, the wake shed from the asymmetric bluff body is easy to be

11 broken as wind flow is strongly separated for the edge of the bluff body, in which the wake intensity

12 would be significantly reduced. As an alternative, we consider to utilize weak magnetic force effect to

13 bring out the internal resonance for an improving energy harvesting performance. In this section, we

14 concentrate on the clarification of synchronization mechanism and evaluation of the improving

15 performance of the magnetically coupled wind energy harvester under internal resonance.

\subsection{Synchronization analysis}

To further validates the analytical solution in the magnetically coupled case, we compared the analytical predictions and numerical solutions by examining the effect of some key parameters on the 
obtained by sweeping the parameters with varying time. Fig. 8 shows the dynamic responses for each oscillator when the detuning parameter changes with fixed associated parameters, in which a very good matching between the analytical predictions and numerical solutions can be observed throughout the concerned range of detuning parameter. This graph shows that a slight amplitude decreasing of the primary oscillator (Fig. 8(a)) corresponding a significant amplitude increase of the secondary oscillator (Fig. 8(b)) as $\sigma$ near to zero, where the 1:1 internal resonance happens. This phenomenon indicates an applied to the bluff body.

energy exchange happens between the two modes of oscillation during their oscillating. It is worth noting that not only stable solutions, but unstable branch of the secondary bluff body were observed in the analytical prediction as shown in Fig. 8(b). The unstable branch is strongly dependent on the wind speed proportional constant $\eta$, in other words, this unstable branch is directly induced by the wind flow

In purpose of representing the dynamic strength distribution over time at various frequencies and examinating the frequency or amplitude synchronizing regime, spectrograms via fast Fourier transformation are adopted as shown in the second row of figure 8. It can be observed that a concentrated and consistent energy distribution of the primary oscillator is located near $20 \mathrm{~Hz}$, and the similar phenomenon can be found in the spectrogram of the secondary oscillator even though there appears some energy distribution in other frequency regimes. This spectrogram indicates that the oscillating frequency of secondary oscillator is locked on that of the primary oscillator. However, secondary natural frequency varies by adjusting detuning parameter $\sigma$, which gives rise to some sub- 
1 harmonic or super-harmonic responses in the secondary oscillators as shown in the spectrogram of

2 secondary oscillator. Obviously, the energy distribution level is higher as $\sigma$ closed to zero, which

3 reflects that the advantages of using 1:1 internal resonance in improving energy conversion efficiency

4 from wind energy to structural vibration energy, and further enhancing the wind energy harvesting

performance.
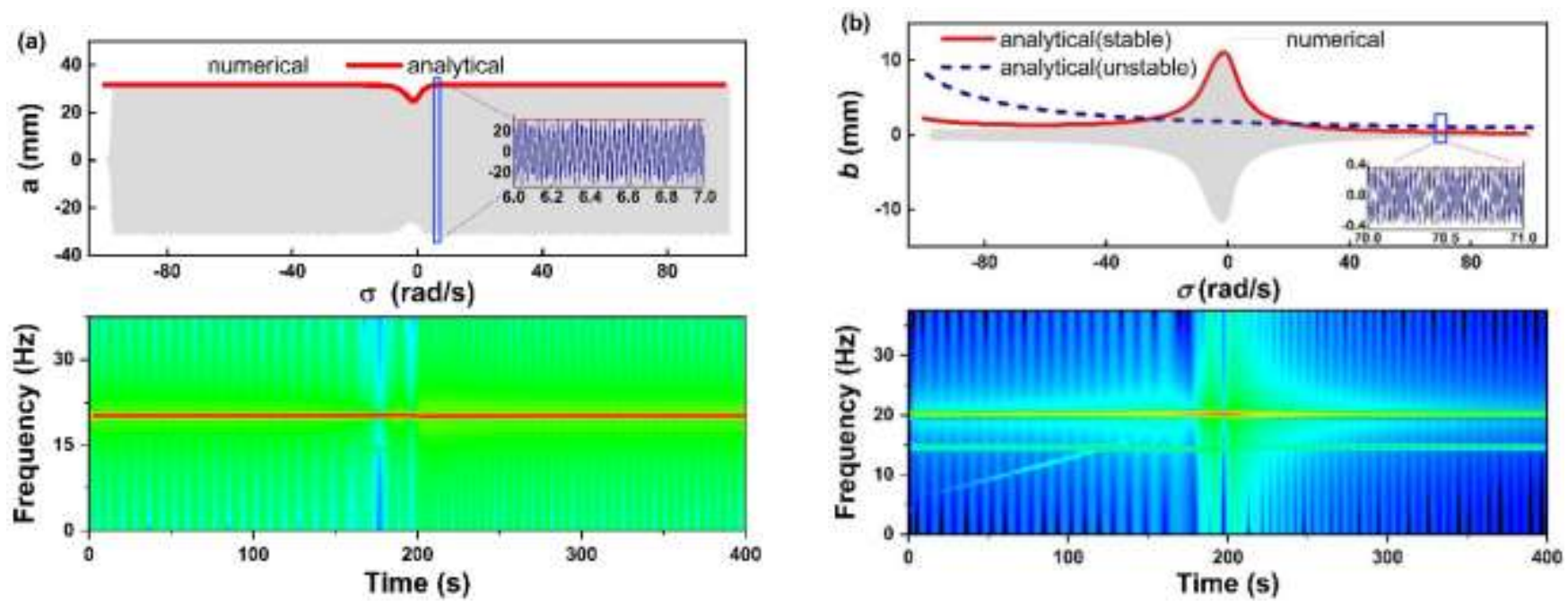

Fig. 8 Dynamic resonances comparisons between analytical results and numerical results versus varying of natural frequency differences and spectrogram for (a) primary oscillator, (b) secondary oscillator and

9 the spectrograms for each oscillator at $D_{m}=20 \mathrm{~mm} \Theta_{w}=0.08, U_{1}=10 \mathrm{~m} / \mathrm{s}$ and $\eta=0.08$.

As the wind speed proportional constant $\eta$ increases to 0.16 , the wind flow induced response curve changes from unstable branch to stable branch as shown Fig. 9 (b). In this case, the dynamic response of

13 the secondary oscillator exhibits a combination of the two stable branches. It also shows that the

14 secondary oscillator amplitude is enlarged as $\sigma$ closed to zero owing to the frequency lock-in phenomenon as shown in the spectrogram of secondary oscillator. Comparing to the case with $\eta=0.08$, 
the increase of the strength of the wind flow applied to the secondary oscillator promotes the occurrence of the galloping induced vibration, and further to be dominant response. It can be observed in the secondary spectrogram that the main energy concentration varies over time following the increasing of $\sigma$ with time variation, which means the secondary oscillator oscillates with itself ascendant natural frequency. Subsequently, some slight changes in oscillating frequency of the primary oscillator happens when $\eta$ increases to 0.16 as shown in the spectrogram of the primary oscillator.
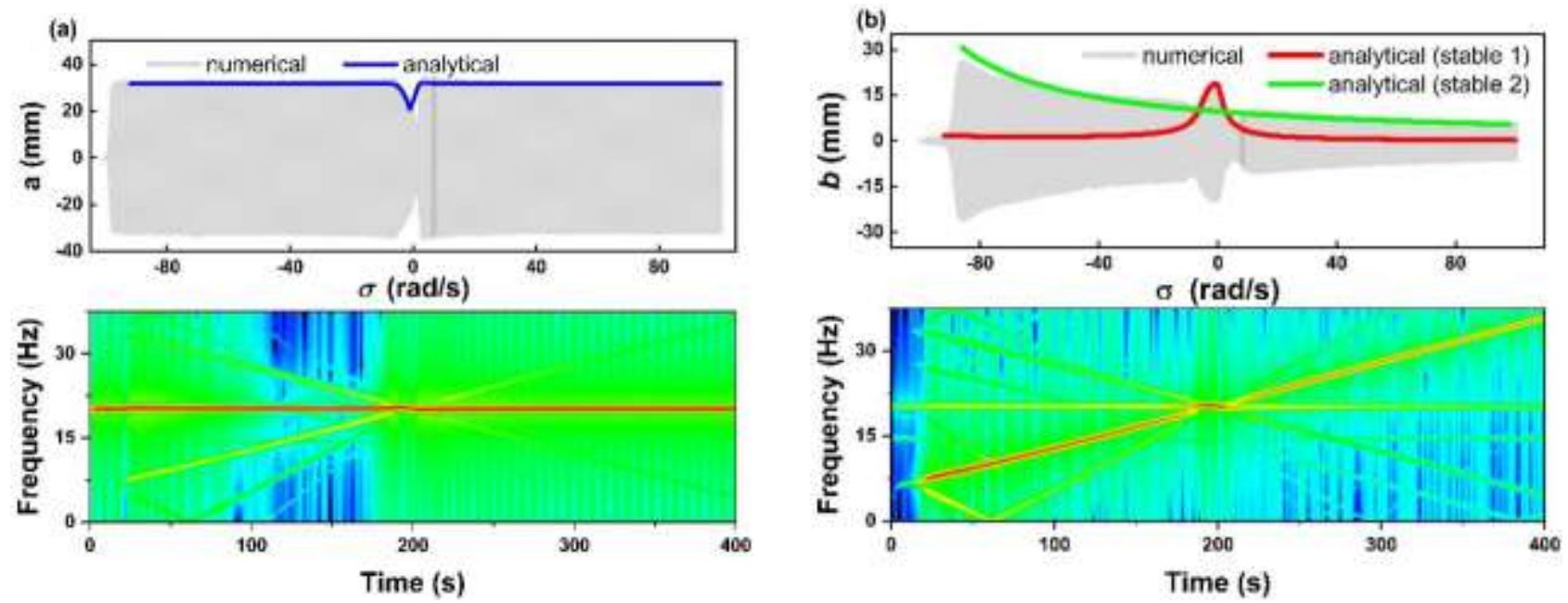

Fig. 9 Dynamic resonances comparisons between analytical results and numerical results versus varying of natural frequency differences and spectrogram analysis for (a) primary oscillator, (b) secondary oscillator and the spectrograms for each oscillator at $D_{m}=20 \mathrm{~mm}, \Theta_{w}=0.08, U_{1}=10 \mathrm{~m} / \mathrm{s}$ at $\eta=0.16$.

As mentioned in the previous section, $\eta$ plays an important role in dynamic response of the secondary oscillator. In a practical situation, $\eta$ is usually a very small value due to the strength deduction of wind flow as experimentally investigated in [35]. Accordingly, it becomes necessary to improve the energy harvesting performance by using magnetic force induced synchronization phenomenon. However, a approximate magnetic force is essential for an induction of synchronization 
because a strongly mode coupling will result in a structural binding phenomenon, which cause the 2-

2

3

4

5

6

7

8

9

10

11

12

13

14

15

16

DOF system collapse to a 1-DOF system. On the other hand, an extremely weak coupling would cause invalidation of mode coupling, and thus make synchronization phenomenon hard to happen. In this study, we investigated the influence of magnet distance to the responses of both oscillators. Subsequently, we investigated the dynamic responses of both the oscillators with a small magnet distance for a strongly mechanical coupling, and the results are illustrated in Fig. 10. It can be observed that both of the primary and secondary oscillators are totally bounded to each other, and they have almost the same dynamic behaviors over the varying frequency range. Based on the prediction accuracy point, it can be found that the analytical prediction results showed in the Fig. 10(a) and (b) does not give an accurate amplitude matching with that of the numerical results. The difference between numerical results and analytical prediction happens because chaos phenomenon happens in this strongly coupled system as detuning parameter $\sigma$ varies, and chaos itself is hard to be predicted. In the spectrogram of each oscillator in second row of Fig. 10, it can be identified that a frequency evolution happens over time, especially, the frequencies evolution is irregular and infinite in the chaos regions. The other regime except the chaos ones also shows a quasi-chaos state even though the dominant frequencies exhibit the same trend of gradual increasing for both of the oscillators. 

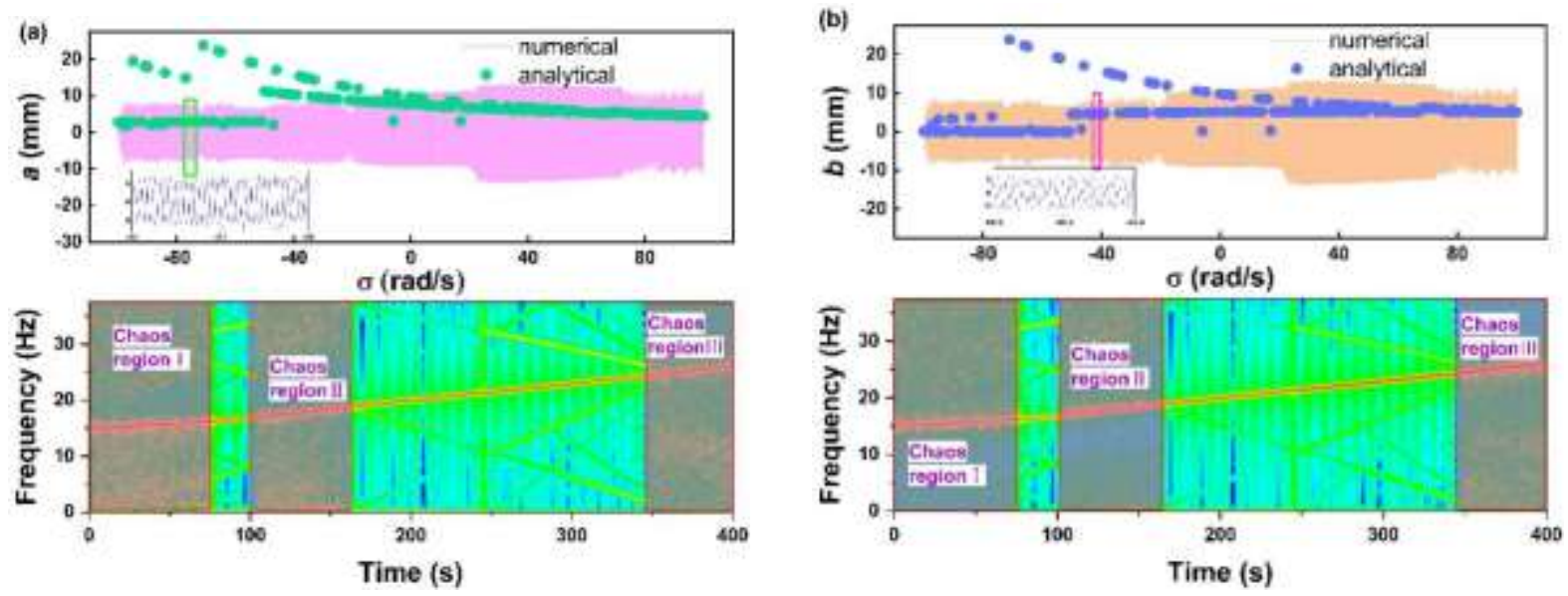

Fig. 10 Dynamic resonances comparisons between analytical results and numerical results versus varying of natural frequency differences for (a) primary oscillator, (b) secondary oscillator and the spectrograms for each oscillator at $\Theta_{w}=0.08, U_{1}=10 \mathrm{~m} / \mathrm{s}, \eta=0.08$ at $D_{m}=5 \mathrm{~mm}$.

To further clarify the influence of the magnetic force strength to the system dynamic responses, we conducted an analytical investigation and compared the dynamic behaviors of the oscillators according to various magnet distances, and the results are shown in Fig. 11. It can be observed that the energy bounded phenomenon happens at the magnet distance of $20 \mathrm{~mm}$, where an amplitude ascending of the primary bluff body corresponds an amplitude descending of the secondary oscillator. Additionally, in this case, the wind flow induced response exhibits unstable. As the magnet distance increases to $40 \mathrm{~mm}$,

11 the energy bounded effect tends to be recessive and a combined dynamic response happens. The wind flow induced response becomes dominant as the magnet distance further increases to $60 \mathrm{~mm}$. In summary, an appropriate magnet distance is significant in improving the effect of energy transform between dynamic modes. 

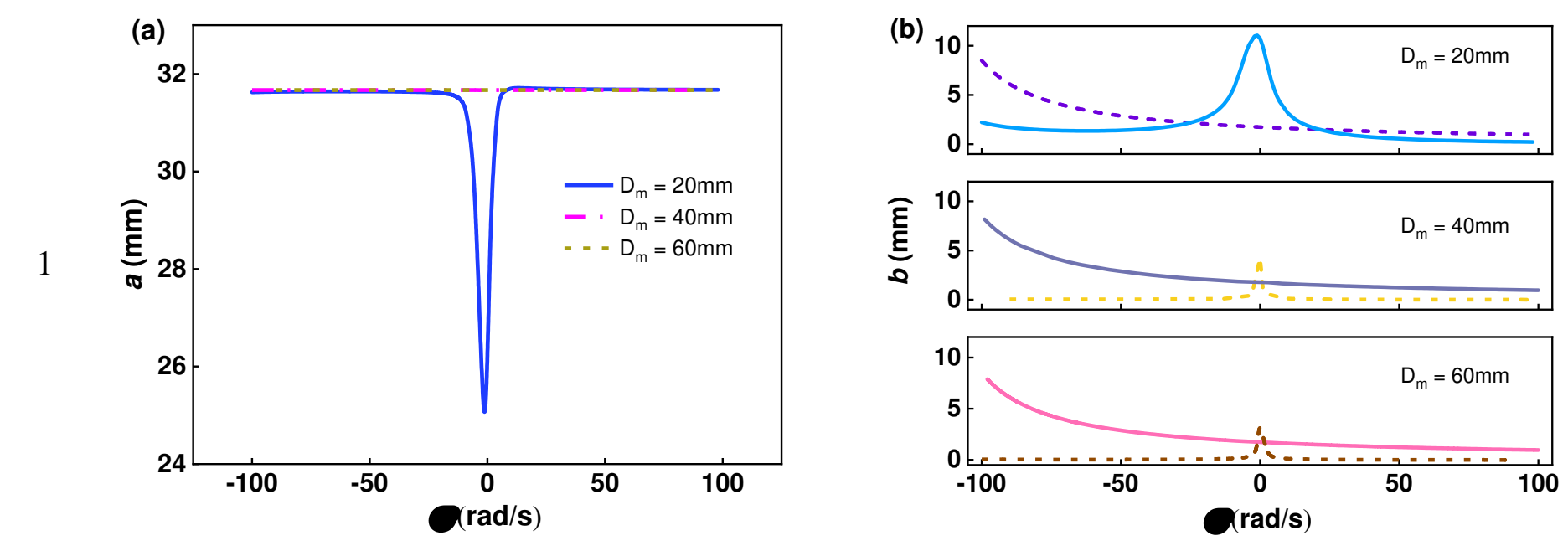

Fig. 11 Dynamic resonances comparisons versus varying of natural frequency differences for (a) primary oscillator, (b) secondary oscillator under different magnet distances of $20 \mathrm{~mm}, 40 \mathrm{~mm}$ and 60 $\mathrm{mm}$.

\subsection{Performance evaluation}

The 1:1 internal resonance is adopted for the mode synchronization between the oscillators, and thus the output performances are expected to be effectively improved. In this study, a maximum power based on an optimal resistance is assumed to evaluate the output performance.

The total RMS voltage are calculated to combine the output voltages from the primary and secondary oscillators according to Eq. (35) with an optimal resistance of $0.18 \mathrm{M} \Omega$. Subsequently, the

11 total maximum average power can be calculated according to Eq. (36). As shown in Fig. 12, the output

12 voltage and average power undergoes a dramatic decrease when $\sigma$ near zero (namely the difference

13 between two natural frequency is closed to zero) at an extremely small magnet distance of $15 \mathrm{~mm}$,

14 because a collapse of degree freedom number happens when the coupling force exceeds a throttle value.

15 It can be seen that total RMS voltage with the maximum value of about $60 \mathrm{~V}$ and average power of 
$10.021 \mathrm{~W}$ with two peaks happens near the position when $\sigma$ is zero as the magnet distance increases to

$220 \mathrm{~mm}$. Subsequently, as we continually increase the magnet distance to $25 \mathrm{~mm}$, two electric output

3 peaks changes to one single peak near the position where $\sigma$ equals zero as shown in Fig. 12.

4 In summary, a significant enhancement of both the total RMS voltage and average power can be

5 achieved by exploiting of the nonlinear magnet force coupling. The 1:1 internal resonance of two

6 oscillators leads to frequency synchronization as natural frequencies of two oscillators are closed to each

7 other, and further promoting the output performance enhancement.

8

9

10

11

12

13

14

15 (a)

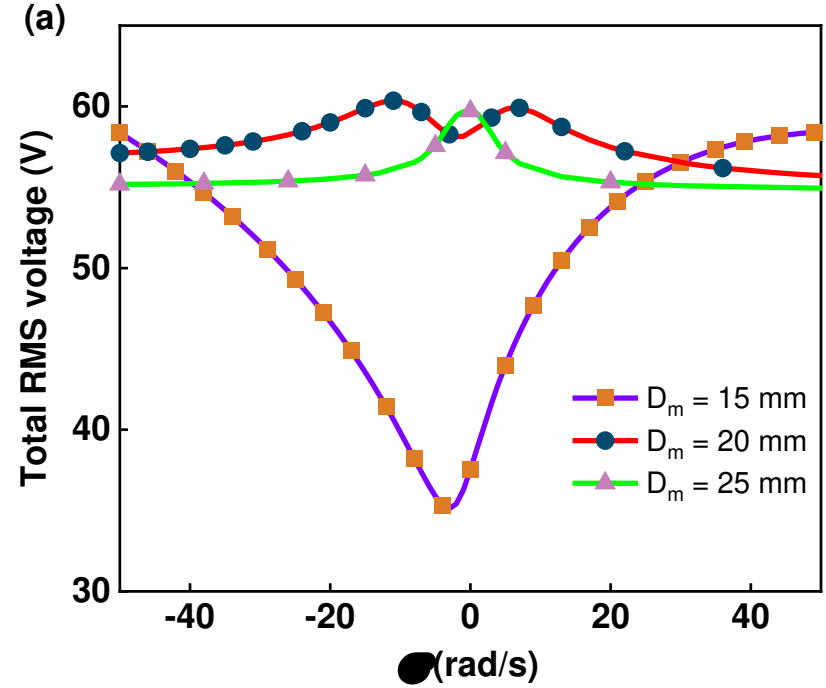

(b)

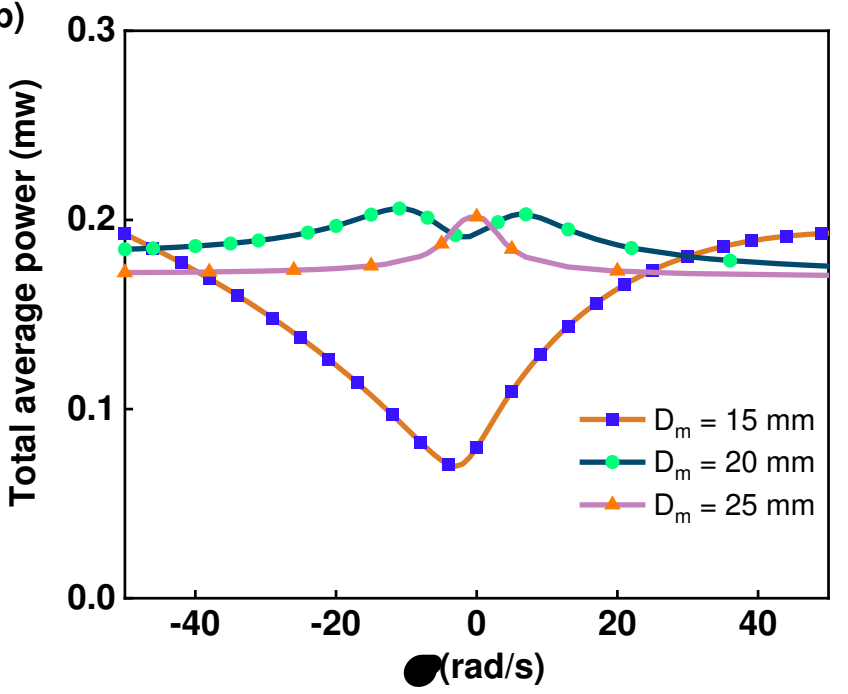

Fig. 12 (a) Total RMS voltages and (b) average powers against various $\sigma$ under different magnet distance of $15 \mathrm{~mm}, 20 \mathrm{~mm}$ and $25 \mathrm{~mm}$.

\section{Conclusions}

In this study, we proposed a technique of 1:1 internal resonance to improve the output performance

in a magnetically coupled wind energy harvester with two tandem oscillators which are arranged along

5 the wind flow direction. The proposed technique was numerical and analytically verified advantageous, 
1 while a significant improvement of total RMS voltage and average power are readily achieved as the

2 frequency synchronization happens when the two natural frequency equals to each other.

The magnetically coupled governing equations representing the transverse dynamics of the bluff bodies and electric circuitry are derived based on the extended Hamilton principle. The oscillating wake generated by the primary bluff body are considered in the proposed model to investigate the dynamic behaviors of both the oscillators. The approximate analytical solutions of the resulting model are

7 determined using a perturbation technique: the method of multiple scales. After mathematical manipulation, a set of modulation equations are obtained to predict the overall static dynamic responses.

9 The proportional wind flow constant $\eta$ is newly defined in this study to describe the wind velocity reduction to the secondary bluff body. A series of parametric studies are carried out to obtain a 11 comprehensive understanding of the relationships between the system dynamic behaviors and the 12 parameters including wind velocity $U_{1}$, frequency detuning parameter $\sigma$ and oscillating wake 13 coefficient $\Theta_{w}$ with absence of magnetic force. The comparisons between the analytical prediction solutions and numerical results are also conducted, which exhibits a very good matching between them. Several saddle-node bifurcation analyses are conducted to reveal the nature of the system with $\eta-\Theta_{w}$ and $\sigma-\Theta_{w}$ plane. The cusp bifurcation and synchronization region are found out based on the bifurcation analyses. The frequency synchronization under magnetic coupling can be triggered as the natural frequencies

19 of the oscillators close to each other under a suitable magnet distance and verified both in analytical 
1 prediction solutions and numerical results. The total RMS voltages and average power generated by both

2 of the oscillators shows a significant improvement when the magnet distance larger than $15 \mathrm{~mm}$ which is

3 benefit to promote the improvement of energy harvesting performance. This study provides an important

4 guideline for the design of a magnetically coupled high-efficiency 2-DOF wind energy harvester using

5 the 1:1 internal mode resonance. It is expected that the proposed method has a potential in designing

6 compact and efficient wind energy harvesters and broad and practical industrial applications such as

7 wireless sensor networks in the future.

\section{Acknowledgments}

10 This work was supported by the National Natural Science Foundation of China (5210051321) and

11 Scientific Research foundation for Talented Scholars of Jiangsu University (5501730000).

\section{Data availability statement}

All data generated or analysed during this study are included in this published article (and its supplementary information files).

15

\section{Conflict of Interest}

The authors declare that they have no conflict of interest.

\section{References}

19 1. Zhang, L., Meng, B., Xia, Y., Deng, Z., Dai, H., Hagedorn, P., et al.: Galloping triboelectric 20 nanogenerator for energy harvesting under low wind speed. Nano Energy. 70, 104477 (2020) 21 https://doi.org/10.1016/j.nanoen.2020.104477 
2. Wang, G., Liao, W.-H., Zhao, Z., Tan, J., Cui, S., Wu, H., et al.: Nonlinear magnetic force and dynamic characteristics of a tri-stable piezoelectric energy harvester. Nonlinear Dynamics. 97(4), 2371-97 (2019) https://doi.org/10.1007/s11071-019-05133-z

3. Maamer, B., Boughamoura, A., Fath El-Bab, A.M.R., Francis, L.A., Tounsi, F.: A review on design improvements and techniques for mechanical energy harvesting using piezoelectric and electromagnetic schemes. Energy Convers Manage. 199, 111973

(2019) https://doi.org/10.1016/j.enconman.2019.111973

4. Dai, J., Tan, Y., Shen, X.: Investigation of energy output in mountain wind farm using multiple-units SCADA data. Appl Energy. 239, 225-38 (2019) https://doi.org/10.1016/j.apenergy.2019.01.207

5. Kaplan, Y.A., Agalar, S., Bildircin, H.: General situation of wind energy source in Turkey and wind turbine technologies. International Journal of Renewable Energy Technology. 10(1-2), 56-67 (2019) https://doi.org/10.1504/IJRET.2019.097004

6. Sun, W., Jang, H., Seok, J.: Magnetically coupled piezoelectric galloping-based energy harvester using a tandem configuration. Mechanical Systems and Signal Processing. 161, 107952 (2021) https://doi.org/10.1016/j.ymssp.2021.107952

7. Hu, G., Wang, J., Tang, L.: A comb-like beam based piezoelectric system for galloping energy harvesting. Mechanical Systems and Signal Processing. 150, 107301 (2021) https://doi.org/10.1016/j.ymssp.2020.107301

8. Tan, T., Hu, X., Yan, Z., Zou, Y., Zhang, W.: Piezoelectromagnetic synergy design and performance analysis for wind galloping energy harvester. Sensors and Actuators A: Physical. 302, 111813 (2020) https://doi.org/10.1016/j.sna.2019.111813

9. Hu, G., Liang, J., Lan, C., Tang, L.: A twist piezoelectric beam for multi-directional energy harvesting. Smart Mater Struct. 29(11), 11 lt01 (2020) https://doi.org/10.1088/1361-665X/abb648

10. Pan, J., Qin, W., Deng, W., Zhang, P., Zhou, Z.: Harvesting weak vibration energy by integrating piezoelectric inverted beam and pendulum. Energy. 227, $120374 \quad$ (2021) https://doi.org/10.1016/j.energy.2021.120374

11. Zhao, L., Tang, L., Yang, Y.: Comparison of modeling methods and parametric study for a piezoelectric wind energy harvester. Smart Mater Struct. 22(12), 125003 (2013) https://doi.org/10.1088/0964-1726/22/12/125003

12. Rezaei-Hosseinabadi, N., Tabesh, A., Dehghani, R.: A Topology and Design Optimization Method for Wideband Piezoelectric Wind Energy Harvesters. IEEE Transactions on Industrial Electronics. 63(4), 2165-73 (2015) https://doi.org/10.1109/tie.2015.2499248

13. Hu, G., Wang, J., Su, Z., Li, G., Peng, H., Kwok, K.C.S.: Performance evaluation of twin piezoelectric wind energy harvesters under mutual interference. Appl Phys Lett. 115(7), 073901 (2019) https://doi.org/10.1063/1.5109457

14. Sun, W., Seok, J.: Novel galloping-based piezoelectric energy harvester adaptable to external wind velocity. Mechanical Systems and Signal Processing. 152, 107477

(2021) https://doi.org/10.1016/j.ymssp.2020.107477

15. Sun, W., Seok, J.: A novel self-tuning wind energy harvester with a slidable bluff body using vortex- 
induced vibration. Energy Convers Manage. 205, 112472

(2020)

https://doi.org/10.1016/j.enconman.2020.112472

16. Zhao, D., Hu, X., Tan, T., Yan, Z., Zhang, W.: Piezoelectric galloping energy harvesting enhanced by topological equivalent aerodynamic design. Energy Convers Manage. 222, 113260 (2020) https://doi.org/10.1016/j.enconman.2020.113260

17. Zhao, K., Zhang, Q., Wang, W.: Optimization of Galloping Piezoelectric Energy Harvester with VShaped Groove in Low Wind Speed. Energies. 12(24), 4619-36 (2019) https://doi.org/10.3390/en12244619

18. Lee, Y.J., Zhou, G., Lua, K.B.: Two-dimensional numerical study of isotoxal-star polygonal cylinders in cross-flow. Journal of Wind Engineering and Industrial Aerodynamics. 188, 125-35 (2019) https://doi.org/10.1016/j.jweia.2019.02.020

19. Wang, J., Tang, L., Zhao, L., Zhang, Z.: Efficiency investigation on energy harvesting from airflows in HVAC system based on galloping of isosceles triangle sectioned bluff bodies. Energy. 172, 1066-78 (2019) https://doi.org/10.1016/j.energy.2019.02.002

20. Zhou, C., Zou, H.-X., Wei, K.-X., Liu, J.: Enhanced performance of piezoelectric wind energy harvester by a curved plate. Smart Mater Struct. 28(12), 125022 (2019) https://doi.org/10.1088/1361$665 \mathrm{X} / \mathrm{ab} 525 \mathrm{a}$

21. Tucker Harvey, S., Khovanov, I.A., Murai, Y., Denissenko, P.: Characterisation of aeroelastic harvester efficiency by measuring transient growth of oscillations. Appl Energy. 268, 115014 (2020) https://doi.org/10.1016/j.apenergy.2020.115014

22. Yang, K., Qiu, T., Wang, J., Tang, L.: Magnet-induced monostable nonlinearity for improving the VIV-galloping-coupled wind energy harvesting using combined cross-sectioned bluff body. Smart Mater Struct. 29(7), 07LT1 (2020) https://doi.org/10.1088/1361-665X/ab874c

23. Wang, J., Gu, S., Zhang, C., Hu, G., Chen, G., Yang, K., et al.: Hybrid wind energy scavenging by coupling vortex-induced vibrations and galloping. Energy Convers Manage. 213, 112835 (2020) https://doi.org/10.1016/j.enconman.2020.112835

24. Yang, X., He, X., Li, J., Jiang, S.: Modeling and verification of piezoelectric wind energy harvesters enhanced by interaction between vortex-induced vibration and galloping. Smart Mater Struct. 28(11), 115027 (2019) https://doi.org/10.1088/1361-665X/ab4216

25. Sun, W., Jo, S., Seok, J.: Development of the optimal bluff body for wind energy harvesting using the synergetic effect of coupled vortex induced vibration and galloping phenomena. International Journal of Mechanical Sciences. 156, 435-45 (2019) https://doi.org/10.1016/j.ijmecsci.2019.04.019

26. Lan, C., Tang, L., Hu, G., Qin, W.: Dynamics and performance of a two degree-of-freedom galloping-based piezoelectric energy harvester. Smart Mater Struct. 28(4), 045018 (2019) https://doi.org/10.1088/1361-665X/ab0852

27. Zhao, L., Tang, L., Yang, Y.: Enhanced piezoelectric galloping energy harvesting using 2 degree-offreedom cut-out cantilever with magnetic interaction. Jpn J Appl Phys. 53(6), 060302 (2014) https://doi.org/10.7567/JJAP.53.060302

28. Wang, J., Geng, L., Yang, K., Zhao, L., Wang, F., Yurchenko, D.: Dynamics of the double-beam 
piezo-magneto-elastic nonlinear wind energy harvester exhibiting galloping-based vibration.

Nonlinear Dynamics. 100(3), 1963-83 (2020) https://doi.org/10.1007/s11071-020-05633-3

29. Yang, W., Towfighian, S.: Internal resonance and low frequency vibration energy harvesting. Smart Mater Struct. 26(9), 095008 (2017) https://doi.org/10.1088/1361-665X/aa791d

30. Clementi, F., Lenci, S., Rega, G.: 1:1 internal resonance in a two d.o.f. complete system: a comprehensive analysis and its possible exploitation for design. Meccanica. 55(6), 1309-32 (2020) https://doi.org/10.1007/s11012-020-01171-9

31. Jiang, W.-A., Ma, X.-D., Han, X.-J., Chen, L.-Q., Bi, Q.-S.: Broadband energy harvesting based on one-to-one internal resonance. Chinese Physics B. 29(10), 100503 (2020) https://doi.org/10.1088/1674-1056/aba5fd

32. Jiang, W., Han, X., Chen, L., Bi, Q.: Improving energy harvesting by internal resonance in a springpendulum system. Acta Mechanica Sinica. 36(3), 618-23 (2020) https://doi.org/10.1007/s10409-02000945-4

33. Pu, D., Wei, X., Xu, L., Jiang, Z., Huan, R.: Synchronization of electrically coupled micromechanical oscillators with a frequency ratio of 3:1. Appl Phys Lett. 112(1), 013503 (2018) https://doi.org/10.1063/1.5000786

34. Cao, D.X., Leadenham, S., Erturk, A.: Internal resonance for nonlinear vibration energy harvesting. The European Physical Journal Special Topics. 224(14-15), 2867-80 (2015) https://doi.org/10.1140/epjst/e2015-02594-4

35. Sun, W., Guo, F., Seok, J.: Development of a novel vibro-wind galloping energy harvester with high power density incorporated with a nested bluff-body structure. Energy Convers Manage. 197, 111880 (2019) https://doi.org/10.1016/j.enconman.2019.111880

36. Lan, C., Tang, L., Qin, W., Xiong, L.: Magnetically coupled dual-beam energy harvester: Benefit and $\begin{array}{lllllll}\text { trade-off. J Intell Mater } \quad \text { Syst } \quad \text { Struct. } & \text { 29(6), } & \text { 1216-35 }\end{array}$ https://doi.org/10.1177/1045389x17730927

37. Meseguer, J., Sanz-Andrés, A., Alonso, G.: Determination of Maximum Mechanical Energy Efficiency in Energy Galloping Systems. J Eng Mech. 141(1), 04014101 (2015) https://doi.org/10.1061/(asce)em.1943-7889.0000817

38. Abdelkefi, A., Yan, Z., Hajj, M.R.: Performance analysis of galloping-based piezoaeroelastic energy 30 harvesters with different cross-section geometries. J Intell Mater Syst Struct. 25(2), $246-56$ (2014) https://doi.org/10.1177/1045389X13491019

39. Tang, L., Zhao, L., Yang, Y., Lefeuvre, E.: Equivalent Circuit Representation and Analysis of Galloping-Based Wind Energy Harvesting. IEEE/ASME Transactions on Mechatronics. 20(2), 834-44 (2015) https://doi.org/10.1109/tmech.2014.2308182

40. Bibo, A., Daqaq, M.F.: An analytical framework for the design and comparative analysis of galloping energy harvesters under quasi-steady aerodynamics. Smart Mater Struct. 24(9), 094006 (2015) https://doi.org/10.1088/0964-1726/24/9/094006 